Annales Henri Lebesgue

3 (2020) 615-648

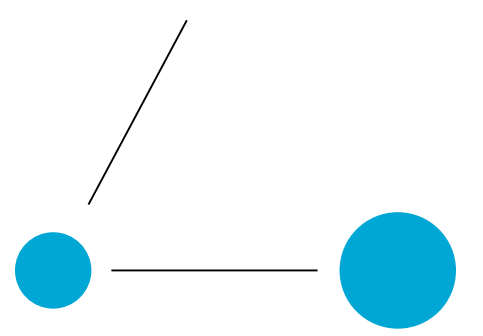

A N N A L E S

HENRI LEBESGUE

\title{
LUBOMIR GAVRILOV
}

ON THE CENTER-FOCUS

PROBLEM FOR THE EQUATION

$\frac{\mathrm{d} y}{\mathrm{~d} x}+\sum_{i=1}^{n} a_{i}(x) y^{i}=0,0 \leqslant x \leqslant 1$

WHERE $a_{i}$ ARE POLYNOMIALS

SUR LE PROBLĖME DU CENTRE-FOYER

POUR L'ÉQUATION

$\frac{\mathrm{d} y}{\mathrm{~d} x}+\sum_{i=1}^{n} a_{i}(x) y^{i}=0,0 \leqslant x \leqslant 1$ OÙ LES $a_{i}$

SONT DES POLYNOMES

ABstract. - We study irreducible components of the set of polynomial plane differential systems with a center, which can be seen as a modern formulation of the classical center-focus problem. The emphasis is given on the interrelation between the geometry of the center set and the Picard-lefschetz theory of the bifurcation (or Poincaré-Pontryagin-Melnikov) functions. Our main illustrative example is the center-focus problem for the Abel equation on a segment, which is compared to the related polynomial Liénard equation.

RÉsumÉ. - Nous étudions les composantes irréductibles de l'ensemble des champs polynomiaux plans avec centre, ce qui peut être vu comme une formulation moderne du problème classique du centre-foyer de Poincaré. L'accent est mis sur l'interrelation entre la géométrie de l'ensemble des centres et la théorie de Picard-Lefschetz des fonctions de bifurcation (ou de Poincaré-Pontryagin-Melnikov). Notre exemple principal est le problème du centre-foyer pour l'équation d'Abel sur un segment, comparée à l'équation de Liénard associée.

Keywords: center-focus problem, Abel equation, Liénard equation. 2020 Mathematics Subject Classification: 37F75, 34C14, 34C05.

DOI: https://doi.org/10.5802/ahl.41

$\left(^{*}\right)$ Research partially supported by the Grant No DN 02-5 of the Bulgarian Fund "Scientific Research". 


\section{The center-focus problem}

The plane differential system

$$
\dot{x}=P(x, y), \quad \dot{y}=Q(x, y)
$$

is said to have a center at the singular point $(0,0)$, if in a sufficiently small neighbourhood of this point all orbits are closed. Consider the scalar differential equation

$$
\frac{\mathrm{d} y}{\mathrm{~d} x}+f(x, y)=0, \quad x \in[0,1]
$$

in which $f(x, 0)=0, \forall x \in[0,1]$. The equation (1.2) is said to have a center at $y=0$, if all solutions $y(x)$ starting near the origin, satisfy $y(0)=y(1)$ (the interval $[0,1]$ can be replaced by any closed interval).

Note on the terminology. We do not specify here the category to which belong $P, Q, f$. They will be either analytic or polynomial, depending on the context. The base field will be either $\mathbb{R}$ or $\mathbb{C}$ depending on the context too. Most results will be valid for both. Thus, the definition of a center for (1.2) is the same in the real and in the complex case. In the case of an analytic complex plane vector field (1.1) the "complex" definition of a center is less straightforward. We say that the origin is a non-degenerate center, if the vector field has an analytic first integral with a Morse critical point at the origin. If this is the case, we shall also say that (1.1) has a Morse singular point, e.g. [CLN96, Dul08]. We recall therefore

Definition 1.1. - The analytic complex vector field (1.1) is said to have a Morse singular point, if it allows an analytic first integral in a neighbourhood of this point, which has a Morse type singularity.

If (1.1) has a Morse singular point, then the linear part of (1.1) is diagonalisable with non-zero eigenvalues, that is to say the singular point of the vector field is non-degenerate.

An example is the saddle $x^{\prime}=x, y^{\prime}=-y$ which has an analytic first integral $x y$ of Morse type, and hence a Morse critical point. Of course, it is linearly equivalent (over $\mathbb{C}$ ) to $x^{\prime}=y, y^{\prime}=-x$ with first integral $x^{2}+y^{2}$ which is the usual linear real center. The advantage to study Morse critical points over $\mathbb{C}$ is that we can use complex analysis and complex algebraic geometry. This is the point of view adopted in these notes.

The two equations (1.1) and (1.2) are closely related. First, a polar change of variables transforms a plane system (1.1) with a center, to equivalent equation of the form (1.2) with a center along the interval $[0,2 \pi]$. Second, if the family of functions $f(\cdot, y), x \in[0,1]$ is replaced by its Fourier series $\widehat{f}(\cdot, y)($ so $\widehat{f}(x+1, y)=\widehat{f}(x, y))$ and the equation (1.2) has a center at $y=0$, then the new system

$$
\frac{\mathrm{d} y}{\mathrm{~d} x}+\widehat{f}(x, y)=0, \quad(x, y) \in \mathbb{R} / \mathbb{Z} \times \mathbb{R}
$$

will have all its orbits starting near the periodic solution $y=0$ on the cylinder $\mathbb{R} / \mathbb{Z} \times \mathbb{R}$, periodic too. Of course, if the smooth function $f$ is non-periodic, then the function $\hat{f}$ is only piece-wise continuous in $x$. The transport map of (1.2) along $[0,1]$ becomes a return map for (1.3) and the definition of a limit cycle for (1.2) 
is straightforward too. Actually, the scalar equation (1.2) in which $f$ is a regular function, should be considered as a simplified model of the eventually singular equation

$$
\frac{\mathrm{d} y}{\mathrm{~d} x}=\frac{P(x, y)}{Q(x, y)}
$$

We resume the above considerations in the following definitions, which make sense both on $\mathbb{R}$ or $\mathbb{C}$ :

DeFinition 1.2. - Let $\varphi=\varphi\left(\cdot ; x_{0}, y_{0}\right)$ be the general solution of the equation $\mathrm{d} y+f(x, y) \mathrm{d} x=0$ with initial condition $y_{0}=\varphi\left(x_{0} ; x_{0}, y_{0}\right)$, on the interval $\left[x_{0}, x_{1}\right]$.

(i) The solution $\varphi=\varphi\left(\cdot ; x_{0}, y_{0}\right)$ is said to be periodic iff $\varphi\left(x_{1} ; x_{0}, y_{0}\right)=y_{0}$

(ii) The solution $\varphi=\varphi\left(\cdot ; x_{0}, y_{0}\right)$ is said to be a limit cycle, provided that it is periodic and isolated, that is to say there is a neighbourhood of its orbit on $S^{1} \times \mathbb{R}$ free of periodic solutions.

(iii) the map $y \mapsto \varphi\left(x_{1} ; x_{0}, y\right)$ is the first return map of (1.2) in a neighbourhood of $y=y_{0}$.

(iv) The equation (1.2) defines a center in a neighbourhood of the periodic solution $\varphi$ provided that the first return map is the identity map in a neighbourhood of $y_{0}$. If the return map is not the identity map, then we say that (1.2) defines a focus at the periodic solution $\varphi$.

The center focus-problem for the equation (1.2) or (1.1) is, roughly speaking, to distinguish between a center and a focus. The algebro-geometric content of the problem is as follows. Suppose, that (1.2) is polynomial, more precisely

$$
\frac{\mathrm{d} y}{\mathrm{~d} x}+\sum_{i=0}^{m} a_{i}(x) y^{i+1}, \quad a_{i} \in \mathbb{C}[x], \operatorname{deg} a_{i} \leqslant n, x \in[0,1] .
$$

The first return map $y \mapsto \varphi(1 ; 0, y)$ is well defined and analytic near the periodic solution $y=0$, and moreover

$$
\varphi(1 ; 0, y)=y+\sum_{n=1}^{\infty} c_{n}(a) y^{n+1}
$$

As we shall see in Theorem 2.3, under the condition $a_{1}=0$, the coefficients $c_{n}=c_{n}(a), n \geqslant 1$, are polynomials in the coefficients of $a_{j}=a_{j}(x), j \leqslant n$. The condition that $\varphi(1 ; 0, \cdot)$ is the identity map determines an infinite number of polynomial relations $\left\{c_{n}(a)=0\right\}$ on the coefficients of the polynomials $a_{j}$. By the Hilbert basis theorem, only a finite number of them are relevant, and they define an algebraic variety (the so called center variety $\mathcal{C}_{m, n}$ ) in the vector space of all coefficients of the polynomials $a_{j}$. The problem is therefore (as formulated by Lins Neto [LN14] in the context of a polynomial foliation induced by (1.1)):

Describe the irreducible components of $\mathcal{C}_{m, n}$. 
The content of the paper is as follows.

In Section 2 we give first an explicit formula for the general solution of the equation

$$
\frac{\mathrm{d} y}{\mathrm{~d} x}+\sum_{i=0}^{n} a_{i}(x) y^{i+1}=0, \quad 0 \leqslant x \leqslant 1
$$

in terms of iterated path integrals, see Theorem 2.1. As a by-product we obtain a formula for the first return map, and explicit center conditions found first by Brudnyi, see Theorem 2.3.

Section 3 is devoted to the perturbation theory of the integrable Abel equation

$$
\frac{\mathrm{d} y}{\mathrm{~d} x}=a(x) y^{2}
$$

with first integral

$$
H=\frac{1}{y}+A(x), \quad A(x)=\int a(x) \mathrm{d} x .
$$

It is assumed that $A(0)=A(1)$, so the equation has a center along [0,1]. We are interested in the number of limit cycles (isolated solutions, such that $y(0)=y(1)$ ) which the perturbed equation $\frac{\mathrm{d} y}{\mathrm{~d} x}=a(x) y^{2}+\ldots$ can have. The center-focus problem for this perturbed equation leads to a well known polynomial moment problem. Under general assumptions this problem has an elegant solution, due to Colin Christopher, Theorem 3.2, which is presented here in the setting of Abelian integrals of dimension zero.

In Section 4 we study irreducible components of the center variety of the polynomial Abel equation (on the interval $[0,1]$ )

$$
\frac{\mathrm{d} y}{\mathrm{~d} x}=p(x) y^{2}+q(x) y^{3}
$$

as well center variety of the related Liénard equation (by "center" we mean the usual Morse center in a neighbourhood of the origin in $\mathbb{C}^{2}$ )

$$
\dot{x}=y, \quad \dot{y}=-q(x)-y p(x) .
$$

In Section 4.1 we prove that the set of Abel equations coming from "pull back" provide irreducible components of the center set, Theorem 4.1. These results are inspired by previous contributions of Movasati.

In Sections 4.2 we revisit the classical center-focus for quadratic vector fields, with special attention to the $Q_{4}$ component of the center set.

In Section 4.3 we give a full description of the center set of Liénard type equations (1.6). These results belong mainly to Cherkas and Christopher, but we present them in the broader context of the present notes. In particular, the base field will be $\mathbb{C}$, see Theorem 4.8 and 4.10 . The centers found in this way are always of "pull back" type. This suggests that the only centers of the related Abel equation (1.5) are of "pull back" type too, which is the content of the so called Composition Conjecture for the Abel equation (1.5) [BRY10, p. 444] to be discussed in Section 4.4. In this last section we show, however, that there are scalar Abel equations with a center along $[0,1]$, which can not be obtained by a "pull back". These equations have a Darboux type first integral, and their construction is inspired by the study of the $Q_{4}$ component in Section 4.2. Among them we find the recent counter-example 
to the Composition Conjecture mentioned above, found first by Giné, Grau and Santallusia [GGS19]. ${ }^{(1)}$

\section{Acknowledgement}

The author is obliged to Jean-Pierre Françoise for the illuminating discussions on the center-focus problem. I thank also the anonymous referees for several suggestions, which helped to improve the text.

\section{The first return map and the Brudnyi formula}

In this section we shall describe the return map of (1.4) as a power series involving iterated path integrals. We prove an explicit formula, due to Brudnyi [Bru06], which amounts to solve the differential equation. The classical approach to do this is by the Picard iteration method. If $y_{0}$ is the initial condition at $x_{0}$ of the differential equation

then the Picard iteration is

$$
\mathrm{d} y=f(x, y) \mathrm{d} x
$$

$$
y_{n+1}(x)=y_{0}+\int_{x_{0}}^{x} y_{n}(t) \mathrm{d} t
$$

where $y_{n}$ tends to the solution of the equation as $n \rightarrow \infty$. We illustrate this on the example $\mathrm{d} y=y \mathrm{~d} x$. If $y_{0}$ is the initial condition at $x=0$ then

$$
\begin{aligned}
& y_{1}(x)=y_{0}+\int_{0}^{x} y_{0} \mathrm{~d} t \\
& y_{2}(x)=y_{0}+\int_{0}^{x} y_{1}(t) \mathrm{d} t=y_{0}+\int_{0}^{x} y_{0} \mathrm{~d} t+\iint_{0 \leqslant t_{2} \leqslant t_{1} \leqslant x} y_{0} \mathrm{~d} t_{1} \mathrm{~d} t_{2} .
\end{aligned}
$$

As

$$
\int \ldots \int_{0 \leqslant t_{n} \leqslant \cdots \leqslant t_{1} \leqslant x} y_{0} \mathrm{~d} t_{1} \ldots \mathrm{d} t_{n}=y_{0} \frac{x^{n}}{n !}
$$

we get $y(x)=y_{0} e^{x}$ as expected. The multiple (or iterated) integrals above appear in a similar way in the non-autonomous linear $\mathrm{d} y=a(x) y \mathrm{~d} x$, or even non-linear case $\mathrm{d} y=f(x, y) \mathrm{d} x$. The non-linear case is more involved, it is reduced to the linear one, but after introducing infinitely many new variables $y, y^{2}, y^{3}, \ldots$ To get around this reduction we shall use a simple Ansatz, for which we need a formal definition of iterated integral.

Let $A s s_{\omega}$ be the graded free associative algebra generated by the infinite dimensional vector space of differential one-forms $\omega=a(x, y) \mathrm{d} x, a \in \mathbb{C}\{x, y\}$. Its elements are non-commutative polynomials in such one-forms. The differential operator

$$
\begin{gathered}
D: A s s_{\omega}^{1} \rightarrow A s s_{\omega}^{1} \\
D(a(x, y) \mathrm{d} x)=\frac{\partial}{\partial y} a(x, y) \mathrm{d} x
\end{gathered}
$$

(1) The present paper is an extended version of two lectures given during the Zagreb Dynamical Systems Workshop, October 22-26, 2018. 
induces a differential operator on $A s s_{\omega}$ which acts by the Leibnitz rule. The readers familiar with the Picard-Lefschetz theory will recognize in $D$ an avatar of the covariant derivative of an Abelian differential on the level sets $\{y=c\}_{c}$.

To save brackets, it is convenient to introduce the following notation

$$
D \omega_{1} \omega_{2} \ldots \omega_{n}=D\left(\omega_{1} \omega_{2} \ldots \omega_{n}\right)
$$

so that (using brackets)

$$
D \omega_{1} \omega_{2}=D\left(\omega_{1} \omega_{2}\right)=\left(D \omega_{1}\right) \omega_{2}+\omega_{1}\left(D \omega_{2}\right) .
$$

and

$$
D \omega_{1} D \omega_{2}=D\left(\omega_{1} D \omega_{2}\right)=\left(D \omega_{1}\right)\left(D \omega_{2}\right)+\omega_{1}\left(D^{2} \omega_{2}\right) .
$$

If we use the notation

$$
D^{k} \omega=\omega^{(k)}
$$

then

$$
D \omega_{1} \omega_{2}=\omega_{1}^{\prime} \omega_{2}+\omega_{1} \omega_{2}^{\prime}
$$

and

$$
D \omega_{1} D \omega_{2}=\left(\omega_{1} \omega_{2}^{\prime}\right)^{\prime}=\omega_{1}^{\prime} \omega_{2}^{\prime}+\omega_{1} \omega_{2}^{\prime \prime} .
$$

For $\omega_{1} \omega_{2} \ldots \omega_{n} \in A s s_{\omega}^{n}, \omega_{k}=\varphi_{k}(x, y) \mathrm{d} x$, define the iterated integral $\int_{x_{0}}^{x} \omega_{1} \omega_{2} \ldots \omega_{n}$ of length $n$, as equal to

$$
\int \ldots \int_{x_{0} \leqslant t_{n} \leqslant \cdots \leqslant t_{1} \leqslant x} \varphi_{1}\left(t_{1}, y\right) \ldots \varphi_{n}\left(t_{n}, y\right) \mathrm{d} t_{1} \ldots \mathrm{d} t_{n} .
$$

The iterated integral allows also a recursive definition (hence the name):

$$
\int_{x_{0}}^{x} \omega_{n} \omega_{n-1} \ldots \omega_{1}=\int_{x_{0}}^{x}\left(\varphi_{n}(t) \int_{x_{0}}^{t} \omega_{n-1} \ldots \omega_{1}\right) \mathrm{d} t
$$

where in the case $n=1$ we have the Riemann integral $\int_{x_{0}}^{x} \omega_{1}$. We note, that the usual notation for the multiple integral (2.2) is $\int_{x_{0}}^{x} \omega_{n} \omega_{n-1} \ldots \omega_{1}$ on the place of $\int_{x_{0}}^{x} \omega_{1} \omega_{2} \ldots \omega_{n}$, see Chen [Che77] or Hain [Hai87]. The reason to prefer the definition (2.3) is that it is better adapted to applications in differential equation, e.g. [Gav05]. Recall in this context, that

$$
\int_{x_{0}}^{x} \omega_{n} \omega_{n-1} \ldots \omega_{1}=(-1)^{n} \int_{x}^{x_{0}} \omega_{1} \omega_{2} \ldots \omega_{n} .
$$

For a short summary of properties of iterated integrals which we use, see [Gav05, Appendix], [GMN09, Section 2].

Theorem 2.1. - With the notation (2.1), a first integral of the differential equation $\mathrm{d} y+f(x, y) \mathrm{d} x=0$ is given by the following recursively defined convergent series

$$
\varphi\left(x_{0} ; x, y\right)=y+\int_{x_{0}}^{x} \omega+\int_{x_{0}}^{x} \omega D \omega+\int_{x_{0}}^{x} \omega D \omega D \omega+\ldots
$$

where

$$
\omega=f(x, y) \mathrm{d} x .
$$

The general solution of (1.2) with initial condition $\left(x_{0}, y_{0}\right)$ is given by

$$
y=\varphi\left(x ; x_{0}, y_{0}\right) \text {. }
$$


Example 2.2. - In the linear case

$$
y^{\prime}+\alpha y=0 \Longleftrightarrow \mathrm{d} y+\alpha y \mathrm{~d} x=0
$$

we obtain

$$
\begin{aligned}
\varphi\left(x_{0} ; x, y\right) & =y\left(1+\alpha \int_{x_{0}}^{x} \mathrm{~d} x+\alpha^{2} \int_{x_{0}}^{x} \mathrm{~d} x \cdot \mathrm{d} x+\ldots\right) \\
& =y\left(1+\alpha\left(x-x_{0}\right)+\alpha^{2} \frac{\left(x-x_{0}\right)^{2}}{2}+\cdots=y e^{\alpha\left(x-x_{0}\right)}\right.
\end{aligned}
$$

and the general solution is

$$
y=\varphi\left(x ; x_{0}, y_{0}\right)=y_{0} e^{\alpha\left(x_{0}-x\right)} .
$$

In the quadratic case

$$
\mathrm{d} y+2 x y^{2} \mathrm{~d} x=0, \omega=2 x y^{2} \mathrm{~d} x
$$

we compute recursively

$$
\begin{aligned}
\int_{x_{0}}^{x} \omega & =\int_{x_{0}}^{x} 2 x y^{2} \mathrm{~d} x=x^{2}-x_{0}^{2} \\
\int_{x_{0}}^{x} \omega D \omega & =\int_{x_{0}}^{x} 2 x y^{2} \mathrm{~d} x .4 x y \mathrm{~d} x=y^{3}\left(x^{2}-x_{0}^{2}\right)^{2} \\
\int_{x_{0}}^{x} \omega D \omega \ldots D \omega & =\left(x^{2}-x_{0}^{2}\right)^{n} .
\end{aligned}
$$

Therefore we get the first integral

$$
\varphi\left(x_{0} ; x, y\right)=y+y^{2}\left(x^{2}-x_{0}^{2}\right)+y^{3}\left(x^{2}-x_{0}^{2}\right)^{2}+\ldots
$$

and the corresponding general solution is

$$
\begin{aligned}
y=\varphi\left(x ; x_{0}, y_{0}\right) & =y_{0}+y_{0}^{2}\left(x_{0}^{2}-x^{2}\right)+y^{3}\left(x_{0}^{2}-x^{2}\right)^{2}+\ldots \\
& =\frac{y_{0}}{1-y_{0}\left(x_{0}^{2}-x^{2}\right)} .
\end{aligned}
$$

Proof of Theorem 2.1. We first verify, that for every fixed $x_{0}$, the function $\varphi\left(x_{0} ; x, y\right)$ is a first integral :

$$
\begin{aligned}
d \varphi\left(x_{0} ; x, y\right)= & \frac{\partial}{\partial x} \varphi\left(x_{0} ; x, y\right) \mathrm{d} x+\frac{\partial}{\partial y} \varphi\left(x_{0} ; x, y\right) \mathrm{d} y \\
= & \omega+\omega \int_{x_{0}}^{x} D \omega+\omega \int_{x_{0}}^{x} D \omega D \omega+\omega \int_{x_{0}}^{x} D \omega D \omega D \omega+\ldots \\
& \quad+\left(1+\int_{x_{0}}^{x} D \omega+\int_{x_{0}}^{x} D \omega D \omega+\int_{x_{0}}^{x} D \omega D \omega D \omega+\ldots\right) \mathrm{d} y \\
= & (\omega+\mathrm{d} y) \frac{\partial}{\partial y} \varphi\left(x_{0} ; x, y\right)=0 .
\end{aligned}
$$

As $\varphi\left(x_{0} ; x_{0}, y_{0}\right)=y_{0}$ then the level set $\left\{(x, y): \varphi\left(x_{0} ; x, y\right)=y_{0}\right\}$ contains both $\left(x_{0}, y_{0}\right)$ and $(x, y)$. By symmetry

$$
y=\varphi\left(x ; x_{0}, y_{0}\right)
$$

is the solution of (1.2) with initial condition $y\left(x_{0}\right)=y_{0}$. The convergency proof is by standard a priori estimates (omitted) 
Note that for fixed $x_{0}, x_{1}$ the two return maps

$$
y \mapsto \varphi\left(x_{1} ; x_{0}, y\right), \quad y \mapsto \varphi\left(x_{0} ; x_{1}, y\right)
$$

are mutually inverse. Therefore $\varphi\left(x_{1} ; x_{0}, \cdot\right)=\mathrm{id}$ if and only if $\varphi\left(x_{0} ; x_{1}, \cdot\right)=\mathrm{id}$. Using Theorem 2.1 we can give explicit center conditions. Assume that

$$
f(x, y)=\sum_{i=1}^{\infty} a_{i}(x) y^{i+1}
$$

and develop the return map $\varphi\left(x_{0} ; x_{1}, y\right)$ as a power series in $y$

$$
\varphi\left(x_{0} ; x_{1}, y\right)=y+\sum_{n=1}^{\infty} c_{n}(a) y^{n+1} .
$$

If we denote, by abuse of notations, $a_{i}=a_{i}(x) \mathrm{d} x$ then we get for the first few coefficients $c_{n}(a)$ (compare to [BRY10, p. 450])

$$
\begin{aligned}
& c_{1}(a)=\int_{x_{0}}^{x_{1}} a_{1} \\
& c_{2}(a)=\int_{x_{0}}^{x_{1}} a_{2}+2 a_{1} a_{1} \\
& c_{3}(a)=\int_{x_{0}}^{x_{1}} a_{3}+2 a_{2} a_{1}+3 a_{1} a_{2}+6 a_{1}^{3} \\
& c_{4}(a)=\int_{x_{0}}^{x_{1}} a_{4}+2 a_{3} a_{1}+3 a_{2}^{2}+4 a_{1} a_{3}+6 a_{2} a_{1}^{2}+8 a_{1} a_{2} a_{1}+12 a_{1}^{2} a_{2}+24 a_{1}^{4}
\end{aligned}
$$

and so on. The general form of the coefficients $c_{n}(a)$ is found immediately from Theorem 2.1. We resume this in the following

Theorem 2.3 (Brudnyi's formula [Bru06]). — The coefficients $c_{n}(a)$ of the first return map (2.5) for the differential equation

$$
\frac{\mathrm{d} y}{\mathrm{~d} x}+\sum_{i=1}^{\infty} a_{i}(x) y^{i+1}=0, x \in\left[x_{0}, x_{1}\right]
$$

are given by the formulae

$$
c_{n}(a)=\sum_{i_{1}+\cdots+i_{k}=n} c_{i_{1}, \ldots, i_{k}} \int_{x_{0}}^{x_{1}} a_{i_{1}} \cdots a_{i_{k}}
$$

where

$$
\begin{aligned}
c_{i_{1}} & =1 \\
c_{i_{1}, i_{2}} & =i_{2}+1 \\
c_{i_{1}, i_{2}, i_{3}} & =\left(i_{3}+1\right)\left(i_{2}+1\right) \\
& \vdots \\
c_{i_{1}, \ldots, i_{k}} & =\left(i_{k}+1\right)\left(i_{k}+i_{k-1}+1\right) \ldots\left(i_{k}+\cdots i_{2}+1\right) .
\end{aligned}
$$

The above formula was deduced first by Brudnyi [Bru06, p. 422] under equivalent form, see also [BRY10, Proposition 2.4] in the case (4.28).

Corollary 2.4. - The equation (1.2) has a center on the interval $\left[x_{0}, x_{1}\right]$ if and only if $c_{n}(a)=0$, for every integer $n \geqslant 1$. 
Example 2.5. - Suppose that the equation

$$
\frac{\mathrm{d} y}{\mathrm{~d} x}+a_{1}(x) y^{2}+a_{2}(x) y^{3}+\cdots=0
$$

has a center on the interval $\left[x_{0}, x_{1}\right]$. Then, using as above the notation $a_{i}=a_{i}(x) \mathrm{d} x$ we have

$$
\begin{aligned}
& c_{1}=\int_{x_{0}}^{x_{1}} a_{1}=0 \\
& c_{2}=\int_{x_{0}}^{x_{1}} a_{2}+2 \int_{x_{0}}^{x_{1}} a_{1}^{2}=0 .
\end{aligned}
$$

The identity

$$
2 \int_{x_{0}}^{x_{1}} a_{1}^{2}=\left(\int_{x_{0}}^{x_{1}} a_{1}\right)^{2}
$$

implies then, that $\int_{x_{0}}^{x_{1}} a_{2}=0$. If we consider more specifically the Abel equation

$$
\frac{\mathrm{d} y}{\mathrm{~d} x}+a_{1}(x) y^{2}+a_{2}(x) y^{3}=0
$$

then taking into consideration that $\int_{x_{0}}^{x_{1}} a_{1}^{3}=0$ and

$$
\int_{x_{0}}^{x_{1}}\left(a_{2} a_{1}+3 a_{1} a_{2}\right)=\int_{x_{0}}^{x_{1}} a_{1} a_{2}=0
$$

we obtain $c_{3}=\int_{x_{0}}^{x_{1}} a_{1} a_{2}$. Therefore a necessary condition for the Abel equation (2.6) to have a center on $\left[x_{0}, x_{1}\right]$ is

$$
\int_{x_{0}}^{x_{1}} a_{1}=0, \quad \int_{x_{0}}^{x_{1}} a_{2}=0, \quad \int_{x_{0}}^{x_{1}} a_{1} a_{2}=0
$$

If we suppose that $a_{1}, a_{2}$ are polynomials of degree at most two, these conditions are also sufficient. The case $\operatorname{deg} a_{1}, a_{2}=3$ can be studied similarly, see [BFY98, BFY99, BFY00].

In general, an obvious sufficient condition to have a center is therefore

$$
\int_{x_{0}}^{x_{1}} a_{i_{1}} \cdots a_{i_{k}}=0, \quad \forall i_{j}, k \geqslant 1 .
$$

Centers with the property (2.8) were called universal in [Bru06].

Consider, more specifically, the following equation with polynomial coefficients $a_{i}$

$$
\mathrm{d} y+\sum_{i=1}^{n} y^{i+1} a_{i}(x) \mathrm{d} x=0, \quad a_{i}(x) \in K[x] .
$$

Theorem 2.6 ([Bru06, Corollary 1.20]). - The polynomial equation (2.9) has an universal center on the interval $\left[x_{0}, x_{1}\right]$, if and only if, it is a pull back of some polynomial equation

$$
\mathrm{d} y=\left(\sum_{i=1}^{n} b_{i}(\xi) y^{i+1}\right) \mathrm{d} \xi, \quad b_{i}(\xi) \in K[\xi] .
$$

via a suitable polynomial map $\xi=\xi(x)$ having the property $\xi\left(x_{0}\right)=\xi\left(x_{1}\right)$.

Not all centers of (2.9) are universal, as discovered recently in [GGS19]. 


\section{Bifurcation functions related to Abel equation and a Theorem of Christopher}

In this section we study the following perturbed Abel differential equation on the interval $[0,1]$

or equivalently

$$
y^{\prime}=a(x) y^{2}-\sum_{j=1}^{\infty} \varepsilon^{j}\left(y^{2} p_{j}(x)+y^{3} q_{j}(x)\right)
$$

$$
\frac{\mathrm{d} y}{y^{2}}=a(x) \mathrm{d} x-\varepsilon \omega_{1}-\varepsilon^{2} \omega_{2}-\ldots
$$

where

$$
\omega_{j}=\left(p_{j}(x)+y q_{j}(x)\right) \mathrm{d} x, \quad a=a(x), \quad p_{j}=p_{j}(x), \quad q_{j}=q_{j}(x)
$$

are polynomials of degree

$$
\operatorname{deg} a=n, \operatorname{deg} p_{j} \leqslant n, \operatorname{deg} q_{j} \leqslant n
$$

and $\varepsilon$ is a small parameter, see [BFY98, BFY99, BFY00, Chr00, Yom03]. For $\varepsilon=0(3.1)$ has a first integral

$$
H(x, y)=\frac{1}{y}+A(x), \quad A(x)=\int a(x) \mathrm{d} x .
$$

Question. - How many limit cycles has the perturbed system (3.1) on the interval $[0,1]$ ?

Recall from the preceding section that a solution $y(x)$ such that $y(0)=y(1)$, is called periodic on $[0,1]$. A limit cycle of $(3.1)$ on $[0,1]$ is therefore an isolated periodic solution on $[0,1]$.

The number of the limit cycles in a compact set are bounded by the number of the zeros of the so called bifurcation function, which we define bellow. A limit cycle which remains bounded when $\varepsilon \rightarrow 0$, tends to a periodic solution of the non perturbed system. If the non-perturbed system $(\varepsilon=0)$ has a periodic solution, then necessarily $A(0)=A(1)$, which already implies that it has a center. For this reason we assume from now on that $A(0)=A(1)=0$, so that

$$
\mathrm{d} y=a(x) y^{2} \mathrm{~d} x \Longleftrightarrow \mathrm{d} H=0
$$

has a center along $0 \leqslant x \leqslant 1$. The perturbed equation can be written

$$
\mathrm{d} H-\varepsilon \omega_{1}-\varepsilon^{2} \omega_{2}-\cdots=0 .
$$

For a solution $y(x)$, let $\mathcal{P}_{\varepsilon}$ be the first return map which sends the initial condition $y_{0}=y(0)$ to $y_{1}=y(1)$. We parameterise $\mathcal{P}_{\varepsilon}$ by $h=\frac{1}{y}=H(0, y)=H(1, y)$ and note that $\mathcal{P}_{\varepsilon}$ is analytic both in $h$ and $\varepsilon$ (close to zero). We have therefore for the first return map

$$
\mathcal{P}_{\epsilon}(h)-h=\varepsilon^{k} M_{k}(h)+O\left(\varepsilon^{k+1}\right), \quad M_{k} \neq 0 .
$$

The function $M_{k}$ is the bifurcation function, associated to the equation (3.1). It is also known as "first non-zero Melnikov function". The reader may compare this 
to (2.4) which is another representation of the first return map, defined for small $y$. As we shall see, the bifurcation function is globally defined. Therefore for every compact set $K,[0,1] \subset K \subset \mathbb{R}^{2}$ and all sufficiently small $|\varepsilon|$, the number of the limit cycles of (3.1) in $K$ is bounded by the number of the zeros of the bifurcation function $M_{k}$ (counted with multiplicity).

$M_{k}$ allows an integral representation

$$
M_{k}(h)=\int_{\{H=h\}} \Omega_{k}
$$

where the integration is along the level set

$$
\{H=h\}=\{(x, y): 1 / y+A(x)=h, 0 \leqslant x \leqslant 1\} .
$$

The differential form $\Omega_{k}$ is computed by the classical Françoise's recursion formula [Fra96, Ili98, Rou98] as follows:

If $k=1$ then $\Omega_{1}=\omega_{1}$, otherwise

$$
\Omega_{m}=\omega_{m}+\sum_{i+j=m} r_{i} \omega_{j}, \quad 2 \leqslant m \leqslant k
$$

and the functions $r_{i}, 1 \leqslant i \leqslant k-1$ are determined successively from the identities $\Omega_{i}=\mathrm{d} R_{i}+r_{i} \mathrm{~d} H$.

Note that neither $r_{i}$ nor $R_{i}$ are uniquely defined. The integrals $M_{i}(h)$ are, however, defined unambiguously.

The first order Melnikov function $M_{1}$ was computed first by Lins Neto [LN80, Section 3], see also [BFY00]. We have

$$
\begin{aligned}
M_{1}(h) & =\int_{\{H=h\}} \omega_{1} \\
& =\int_{\{H=h\}} p_{1}(x) \mathrm{d} x+y q_{1}(x) \mathrm{d} x \\
& =\int_{0}^{1} p_{1}(x) \mathrm{d} x+\int_{0}^{1} \frac{q_{1}(x)}{h-A(x)} \mathrm{d} x \\
& =\int_{0}^{1} p_{1}(x) \mathrm{d} x+\sum_{k=0}^{\infty} h^{-k-1} \int_{0}^{1} q_{1}(x) A^{k}(x) \mathrm{d} x .
\end{aligned}
$$

$M_{1}$ vanishes identically if and only if $\int_{0}^{1} p_{1}(x) \mathrm{d} x=0$ and

$$
\int_{0}^{1} q_{1}(x) A^{k}(x) \mathrm{d} x=0, \quad k=0,1,2, \ldots
$$

which is the content of the famous polynomial moment problem for $q_{1}$ and $A$, solved in full generality by Pakovich and Muzychuk [PM09], see also [BFY98, BFY99, BFY00, Chr00, Yom03]. If $M_{1}=0$ by the above formula we get

$$
M_{2}(h)=\int_{\{H=h\}} r_{1} \omega_{1}+\int_{\{H=h\}} \omega_{2}
$$

where $r_{1}$ is computed from the identity $\omega_{1}=\mathrm{d} R_{1}+r_{1} \mathrm{~d} H$. As $\mathrm{d} \omega_{1}=\mathrm{d} r_{1} \wedge \mathrm{d} H$ then $\mathrm{d} r_{1}=\omega_{1}^{\prime}=\frac{\mathrm{d} \omega_{1}}{\mathrm{~d} H}$ is the Gelfand-Leray form of $\omega_{1}$. From the identity $H(x, y(x, h)) \equiv h$ 
we have $\frac{\partial y}{\partial h}=-y^{2}$ and hence

$$
r(x, y)=\int_{0}^{x} \omega_{1}^{\prime}=-\int_{0}^{x} y^{2} q(x) \mathrm{d} x .
$$

We conclude

Proposition 3.1 ([Gav05, Formula (2.8)]). - Under the hypothesis $M_{1}=0$ the second Melnikov function is given by the following iterated integral of length two

$$
M_{2}(h)=\int_{\{H=h\}} \omega_{1} \omega_{1}^{\prime}+\int_{\{H=h\}} \omega_{2}
$$

where

$$
\omega_{1}=p_{1}(x) \mathrm{d} x+y q_{1}(x) \mathrm{d} x, \quad \omega_{1}^{\prime}=-y^{2} q_{1}(x) \mathrm{d} x, \quad \omega_{2}=p_{2}(x) \mathrm{d} x+y q_{2}(x) \mathrm{d} x .
$$

The hypothesis $M_{1}=0$ is of interest for us, as it will allow to compute the tangent space to the center set at the point $(a, 0)$, see the next Section 4 . For our purposes the polynomial $a(x)$ can be taken in a general position, in which case the polynomial moment problem for $q(x), A(x)$ has the following elegant solution

Theorem 3.2 ([Chr00]). - Assume that A, $q$ are complex univariate polynomials, such that

$$
A(0)=A(1)=0, \quad A^{\prime}(0) \neq 0, \quad A^{\prime}(1) \neq 0 .
$$

The multivalued transcendental function

$$
I(h)=\int_{0}^{1} \frac{q(x)}{h-A(x)} \mathrm{d} x
$$

vanishes identically, if and only if the polynomials $Q=\int q$ and $A$ satisfy the following "Polynomial Composition Condition" (PCC):

There exist polynomials $\widetilde{Q}, \widetilde{A}, W$, such that

$$
A=\widetilde{A} \circ W, \quad Q=\widetilde{Q} \circ W, \quad W(0)=W(1) .
$$

Before recalling the proof of Christopher, we put $I$ in the broader context of the Picard-Lefschetz theory.

The function $I(h)$ is well defined for sufficiently big $h$, and has an analytic continuation in a complex domain to certain multivalued function. It is in fact an Abelian integral depending on a parameter. More precisely, consider the genus zero affine curve

$$
\Gamma_{h}=\left\{(x, y) \in \mathbb{C}^{2}: \frac{1}{y}+A(x)=h\right\}, \quad A(0)=A(1)=0 .
$$

It is a Riemann sphere with $n+2$ removed points, provided that $h \neq 0$. The removed points correspond to $\left(x=x_{i}(h), y=\infty\right)$, where $A\left(x_{i}(h)\right) \equiv h$, and to $(x=\infty, y=0)$. Given a divisor $m=P_{0}+P_{1}$ on $\Gamma_{h}$, where

$$
P_{0}=\left(0, \frac{1}{h}\right), \quad P_{1}=\left(1, \frac{1}{h}\right)
$$

we define a singularized algebraic curve $\Gamma_{h}^{\text {sing }}$, see Figure 3.1 .

As a topological space it is just the curve $\Gamma_{h}$ with the two points $P_{0}$ and $P_{1}$ identified to a point $m$. The structural sheaf of $\Gamma_{h}^{\text {sing }}$ is the same as the structural 


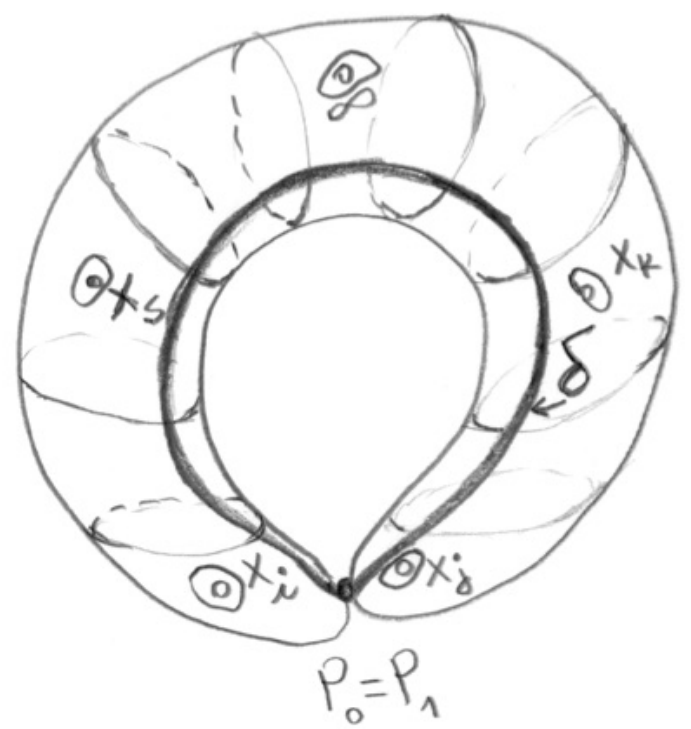

Figure 3.1. The singularized algebraic curve $\Gamma_{h}^{\text {sing }}$.

sheaf of $\Gamma_{h}$, except at the point $m \in \Gamma_{h}^{\text {sing }}$. A function $f$ on $\Gamma_{h}^{s i n g}$ is said to be regular, if it is regular on $\Gamma_{h}$, and moreover $f\left(P_{0}\right)=f\left(P_{1}\right)$. The path $[0,1]$ connecting the points $x=0$ and $x=1$ closes on the singular algebraic curve $\Gamma_{h}^{\text {sing }}$. The function $I(h)$ defined in (3.6) is an Abelian integral on $\Gamma_{h}^{\text {sing }}$

$$
I(h)=\int_{\delta(h)} y q(x) \mathrm{d} x, \quad y=\frac{1}{h-A(x)}
$$

where $\delta(h) \in H_{1}\left(\Gamma_{h}^{\text {sing }}, \mathbb{Z}\right)$ is represented by the closed loop on $\Gamma_{h}^{\text {sing }}$ corresponding to the interval $[0,1]$.

We note that given an arbitrary effective divisor $m=P_{0}+P_{1}+\ldots P_{k}$ on $\Gamma_{h}$, one constructs in a similar way a singularized curve $\Gamma_{h}^{\text {sing }}$, which is the natural framework of the generalized center problem for the Abel equation, see [BFY99, Conjecture 1.7] and [BRY10].

Proof of Theorem 3.2. - The homology group $H_{1}\left(\Gamma_{h}^{s i n g}, \mathbb{Z}\right)$ is of dimension $n+2$. It is generated by $n+1$ simple closed loops $\gamma_{i}=\gamma_{i}(h)$ which make one turn around the $n+1$ punctures $x_{i}(h)$ on $\Gamma_{h}, A\left(x_{i}(h)\right)-h=0$, as well the loop $\delta(h)$ connecting 0 and 1 on the singularized curve $\Gamma_{h}^{\text {sing }}$. The monodromy of the loop $\delta(h)$ is shown on the Figure 3.2.

As the integral $I(h)$ is constant, it follows that

$$
\int_{\gamma_{i}(h)-\gamma_{j}(h)} y q(x) \mathrm{d} x \equiv 0
$$



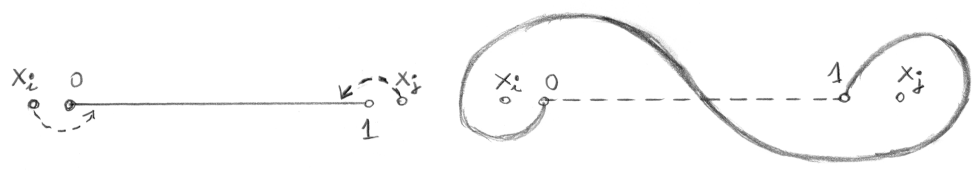

Figure 3.2. The monodromy of $x_{i}(h), x_{j}(h)$ and the loop $\delta(h)$.

where $A\left(x_{i}(0)\right)=A\left(x_{j}(0)\right)=0$ and $x_{i}(0)=0, x_{j}(0)=1$. We have also

$$
\begin{aligned}
\int_{\gamma_{i}(h)-\gamma_{j}(h)} y q(x) \mathrm{d} x & =\int_{\gamma_{i}(h)-\gamma_{j}(h)} \frac{q(x)}{h-A(x)} \mathrm{d} x \\
& =-2 \pi i\left(\frac{q\left(x_{i}(h)\right)}{A^{\prime}\left(x_{i}(h)\right)}-\frac{q\left(x_{j}(h)\right)}{A^{\prime}\left(x_{j}(h)\right)}\right) \\
& =-2 \pi i\left[q\left(x_{i}(h)\right) x_{i}^{\prime}(h)-q\left(x_{j}(h)\right) x_{j}^{\prime}(x)\right] \\
& =-2 \pi i \frac{\mathrm{d}}{\mathrm{d} h}\left[Q\left(x_{i}(h)\right)-Q\left(x_{j}(h)\right)\right]
\end{aligned}
$$

where

$$
Q(x)=\int q(x) \mathrm{d} x
$$

is a primitive of $q$, and $x_{i}(h)$ are the roots of the polynomial $A(x)-h$ (we used that $\left.A^{\prime}\left(x_{i}(h)\right) x_{i}^{\prime}(h) \equiv 1\right)$. We denote,

$$
J(h)=\int_{x_{i}(h)-x_{j}(h)} Q=Q\left(x_{i}(h)\right)-Q\left(x_{j}(h)\right)
$$

and call $J$ an Abelian integral of dimension zero along the zero-cycle

$$
x_{i}(h)-x_{j}(h) \in H_{0}(\{A(x)=h\}, \mathbb{Z})
$$

([GM07, Definition 1]). If the Abelian integral $I(h)$ vanishes identically, then the same holds true for $J^{\prime}(h)$, hence $J(h)=$ const. and it is easy to check that the constant is zero, $J(h) \equiv 0$.

The set of rational functions $Q$ such that $Q\left(x_{i}(h)\right) \equiv Q\left(x_{j}(h)\right.$ is a subfield of the field of all rational functions $\mathbb{C}(x)$. By the Lüroth theorem this subfield is of the form $\mathbb{C}(W)$ for suitable rational function $W$. It follows that $Q=\widetilde{Q} \circ W, A=\widetilde{A} \circ W$ where $\widetilde{Q}, \widetilde{A}, W$ are rational functions. As $Q$ is a polynomial, then $Q^{-1}(\infty)=\{\infty\}$ which implies that $\widetilde{Q}^{-1}(\infty)=\{p\}$ for some $p \in \mathbb{P}^{1}$ and also $W^{-1}(p)=\{\infty\}$, and similarly $\widetilde{A}^{-1}(\infty)=\{p\}$ Let $\varphi$ be a Mobius functions such that $\varphi(p)=\infty$. Then the functions

$$
\widetilde{Q} \circ \varphi^{-1}, \varphi \circ W, \widetilde{A} \circ \varphi^{-1}
$$

are polynomials. For this reason we may suppose that $\widetilde{Q}, \widetilde{A}, W$ are polynomials. If $W\left(x_{i}(h)\right) \equiv W\left(x_{j}(h)\right)$, then clearly $W(0)=W(1)$ and the Theorem 3.2 is proved. 
If $W\left(x_{i}(h)\right) \not \equiv W\left(x_{j}(h)\right)$, then we denote $w_{i}(h)=W\left(x_{i}(h)\right), w_{j}(h)=W\left(x_{j}(h)\right)$ and note that

$$
\widetilde{A}\left(w_{i}(h)\right) \equiv A\left(w_{j}(h)\right) \equiv h, \widetilde{Q}\left(w_{i}(h)\right)=\widetilde{Q}\left(w_{j}(h)\right) .
$$

We may reason then by induction on $\widetilde{A}, \widetilde{Q}$ which have a smaller degree than $A, Q$ respectively. Thus this process must stop and we get $W$ with $W\left(x_{i}(h)\right) \equiv W\left(x_{j}(h)\right)$, and hence $W(0)=W(1)$.

\section{Irreducible components of the Center set}

An affine algebraic variety $V$ in $\mathbb{C}^{n}$ is the common zero locus of a finite collection of polynomials $f_{i} \in \mathbb{C}\left[z_{1}, \ldots, z_{n}\right]$. The variety $V$ is said to be irreducible, if for any pair of closed varieties $V_{1}, V_{2}$ such that $V=V_{1} \cup V_{2}$, either $V_{1}=V$ or $V_{2}=V$. Of course, it might happen that a variety $V$ is reducible $V=V_{1} \cup V_{2}$, where $V_{1}, V_{2} \neq V$. In this case we may ask whether $V_{1}$ and $V_{2}$ are further reducible and so on. It is a basic fact of commutative algebra that in this way only a finitely many irreducible subvarieties $V_{i} \subset V$ can be found, and more precisely:

Any variety $V$ can be uniquely expressed as a finite union of irreducible varieties $V_{i}$ with $V_{i} \varsubsetneqq V_{j}$ for $i \neq j$, e.g. [Har95].

The varieties $V_{i}$ which appear in the finite decomposition

$$
V=\cup_{i} V_{i}
$$

are the irreducible components of $V$.

Let $W \subset V$ be another algebraic variety. Is $W$ an irreducible component of $V$ ? It is usually easy to verify, whether $W$ is irreducible. It is much harder to check that $W$ is an irreducible component of $V$. Indeed, it might happen that $W \varsubsetneqq V_{i}$ where $V_{i}$ is an irreducible component of $V$. To verify this, one may compare the dimensions of the tangent spaces $T_{x} W$ and $T_{x} V$ at some smooth point $x \in V \cap W$ (one point $x$ is enough!). Then $W \varsubsetneqq V_{i}$ if and only if $T_{x} W \varsubsetneqq T_{x} V$. Of course, there might be no way to know that $x$ is a smooth point, in which case we use the tangent cones $T C_{x} W$ and $T C_{x} V$. For every $x \in W$ on an irreducible variety $W$ holds $\operatorname{dim} T C_{x} W=\operatorname{dim} W$. Thus, for irreducible varieties $W \subset V$ holds

$$
\operatorname{dim} T C_{x} W<\operatorname{dim} T C_{x} \Longleftrightarrow W \varsubsetneqq V .
$$

The choice of $x \in W$ is irrelevant, which allows a great flexibility.

The above observation will be applied in the case when $V$ is the center set of the equation (1.2), and $W$ is a subset of equations with a center. In the planar case (1.1) this approach was developed by Movasati [Mov04]. He observed that the vanishing of the first Melnikov function, related to one-parameter deformations (arcs) of systems (1.1) with a center, provides equations for the tangent space $T_{x} W$, while the vanishing of the second Melnikov function provides equations for the tangent cone $T C_{x} W$. This remarkable connection between algebraic geometry and dynamics will allow us to go farther in the description of irreducible components of the center 
set. We adapt the approach of Movasati [Mov04] and Zare [Zar19] to (1.2) in the context of the set $\mathcal{A}_{n}$ of Abel differential equations

$$
\frac{\mathrm{d} y}{\mathrm{~d} x}=a(x) y^{2}+b(x) y^{3}
$$

parameterised by the polynomials $a(x), b(x)$ of degree at most $n$. They form therefore a vector space of dimension $2 n+2$, and consider the subset $\mathcal{C}_{n} \subset \mathcal{A}_{n}$ of Abel differential equations having a center on the interval $0 \leqslant x \leqslant 1$. As we saw in the preceding section, $\mathcal{C}_{n}$ is defined by finitely many polynomial relations $c_{n}(a, b)=0$ and therefore is an algebraic set.

\subsection{Universal centers of the Abel equation define irreducible components of the center set}

If the integer $k>1$ divides $n+1$, then we denote by $\mathcal{U}_{n / k} \subset \mathcal{C}_{n} \subset \mathcal{A}_{n}$ the algebraic closure of the set of pairs of polynomials $(a, b)$ (or Abel equations (4.1)), such that the following Polynomial Composition Condition (PCC) is satisfied

There exist polynomials $\widetilde{A}, \widetilde{B}, W$ of degrees $(n+1) / k,(n+1) / k, k$, such that

$$
A=\widetilde{A} \circ W, \quad B=\widetilde{B} \circ W, \quad W(0)=W(1) .
$$

The differential form associated to (4.1)

$$
\mathrm{d} y-\left(a(x) y^{2}+b(x) y^{3}\right) \mathrm{d} x=\mathrm{d} y-y^{2} \mathrm{~d} A(x)-y^{3} \mathrm{~d} B(x)
$$

is a pull back of the differential form

$$
\mathrm{d} y-\left(\widetilde{A}^{\prime}(w) y^{2}+\widetilde{B}^{\prime}(w) y^{3}\right) \mathrm{d} w=\mathrm{d} y-y^{2} \mathrm{~d} \widetilde{A}(w)-y^{3} \mathrm{~d} \widetilde{B}(w)
$$

under the map $(x, y) \rightarrow(w, y)$, where $w=W(x)$. In other words the equation (4.1) is obtained from

$$
\frac{\mathrm{d} y}{\mathrm{~d} w}=\widetilde{A}^{\prime}(w) y^{2}+\widetilde{B}^{\prime}(w) y^{3}
$$

via the substitution $w=W(x)$. This, combined to $W(0)=W(1)$ implies that the set of Abel equations $\mathcal{U}_{n / k}$ have a center at $y=0$ along $[0,1]$. Of course one could check directly that the center conditions $c_{n}(a)=0$ are satisfied for all $n$ (Theorem 2.3). Indeed, the iterated integrals $\int_{x_{0}}^{x_{1}} a_{i_{1}} \cdots a_{i_{k}}$ vanish, because they are pull backs under $W$ of iterated integrals along an interval, contractible to the point $W\left(x_{0}\right)=W\left(x_{1}\right)$. Following Brudnuyi [Bru06], we say that (4.1) determines an universal center if and only if

$$
\int_{x_{0}}^{x_{1}} a_{i_{1}} \cdots a_{i_{k}}=0, \quad \forall i_{j} \in \mathbb{N} .
$$

It is shown then that a center is universal, if and only if the corresponding equation (4.1) is a pull back under an appropriate polynomial as above, see Brudnyi [Bru06, Corollary 1.20]. Thus, the universal centers are exactly those, obtained by a polynomial pull back in the sense (4.2), see the Polynomial Composition Condition (PCC). 
Note that the universal center set $\mathcal{U}_{n / k}$ is an irreducible algebraic variety, as a Zariski open subset of it is parametrized by the polynomials $\widetilde{A}, \widetilde{B}, W$ of degrees respectively $(n+1) / k,(n+1) / k, k$. The main result of the section is

TheOREM 4.1. - The algebraic sets $\mathcal{U}_{n / k}$ are irreducible components of the center set $\mathcal{C}_{n}$ of the Abel equation

$$
\frac{\mathrm{d} y}{\mathrm{~d} x}=a(x) y^{2}+b(x) y^{3}, \quad \operatorname{deg} a, \operatorname{deg} b \leqslant n .
$$

We shall illustrate first the idea of the proof of Theorem 4.1 on the rather elementary case $k=n+1$. The closure of the universal center set $\mathcal{U}_{n / n+1}$ consists of Abel equations (4.1) such that

$$
\operatorname{deg} a, \operatorname{deg} b \leqslant n, \quad \int_{0}^{1} a(x) \mathrm{d} x=\int_{0}^{1} b(x) \mathrm{d} x=0
$$

and moreover the polynomials $a(x), b(x)$ are co-linear. Thus, $\mathcal{U}_{n / n+1}$ is identified to the vector space of pairs of polynomials $(a(x), b(x))$ with the above properties, and is therefore of dimension $n+1$. Consider now the point $(a(x), 0) \in U_{n / n+1}$ where $a(x)$ is a degree $n$ polynomial.

Proposition 4.2. - The tangent space $T_{(a, 0)} \mathcal{U}_{n / n+1}$ is a vector space of dimension $n+1$, which consists of pairs of polynomials $(p, q)$ of degree at most $n$, such that $q$ and $a$ are co-linear polynomials, and $\int_{0}^{1} p(x) \mathrm{d} x=0$.

The proof is left to the reader. Next, we compute the tangent cone $T C_{(a, 0)} \mathcal{C}_{n}$ at $(a, 0)$ to the center set $\mathcal{C}_{n}$. To avoid complications, we choose $a$ to be a non-composite polynomial.

Proposition 4.3. - Lat a be a non-composite polynomial of degree $n$, such that $a(0) \neq 0, a(1) \neq 0$. Then

$$
T C_{(a, 0)} \mathcal{C}_{n}=T_{(a, 0)} \mathcal{U}_{n / n+1}
$$

The above implies that the algebraic set $\mathcal{U}_{n / n+1}$ is an irreducible component of the center set $\mathcal{C}_{n}$.

Proof of Proposition 4.3. - Consider a one-parameter deformation

$$
\varepsilon \rightarrow(a-\varepsilon p+\ldots,-\varepsilon q+\ldots)
$$

of (4.1) at the point $(a, 0)$. For $\varepsilon=0$ the equation is

$$
\frac{\mathrm{d} y}{y^{2}}=a(x) \mathrm{d} x
$$

and has a first integral $H(x, y)=\frac{1}{y}+A(x)$ where $A$ is a primitive of $a, A(0)=A(1)$. The perturbed equation is

$$
\mathrm{d} H-\varepsilon[p(x)+y q(x)] \mathrm{d} x+\cdots=0 .
$$

We parameterize the cross-sections $\{x=0\},\{x=1\}$ by $h=H(0, y)=H(1, y)=1 / y$ and write for the return map $\varphi_{\varepsilon}$

$$
\varphi_{\varepsilon}(h)=h+\varepsilon M_{1}(h)+O\left(\varepsilon^{2}\right) .
$$


The Melnikov function $M_{1}$, according to Section 3, is computed to be

$$
M_{1}(h)=\int_{\{H=h\}} p(x) \mathrm{d} x+y q(x) \mathrm{d} x=\int_{0}^{1} p(x) \mathrm{d} x+\int_{0}^{1} \frac{q(x)}{h-A(x)} \mathrm{d} x .
$$

Assuming that for all sufficiently small $\varepsilon$ the deformed Abel equation belongs to the center set $\mathcal{C}_{n}$, implies $M_{1}=0$, which on its turn imposes rather severe conditions on the polynomials $p, q$. First, $\int_{0}^{1} p(x) \mathrm{d} x=0$ as follows already from $(2.7)$. The second condition

$$
\int_{0}^{1} \frac{q(x)}{h-A(x)} \mathrm{d} x \equiv 0
$$

is well studied in a number of articles, and is known as the polynomial moment problem, e.g. [BRY10] and the references there. For the case of a general $A$, see the Addendum by Pakovich in [Yom03]. As $a(0) \neq 0, a(1) \neq 0$ then by Theorem 3.2 we have that $\int_{0}^{1} \frac{q(x)}{h-A(x)} \mathrm{d} x \equiv 0$ if and only if the composition condition holds true. As $A$ is supposed to be prime, this means that $A$ and $Q=\int q$ are co-linear polynomials. This completes the proof of Proposition 4.3 and Theorem 4.1 in the case $k=n$.

Note that in full generality, a vector $(p, q)$ which belongs to the tangent cone is a vector, such that there is a one-parameter deformation

$$
\varepsilon \rightarrow\left(a+\varepsilon^{k} p+\ldots, \varepsilon^{k} q+\ldots\right)
$$

at the point $(a, 0)$ which belongs to the center set $\mathcal{C}_{n}$. The same arguments give the same constraints to the vector $(p, q)$.

Proof of Theorem 4.1 in the general case. - Assume that the integer $k>1$ divides $n+1$ and consider the algebraic set $\mathcal{U}_{n / k}$ of Abel differential equations, at $y=0$ along $[0,1]$. The proof follows the same lines as the case $k=n$, with the notable difference that the second Melnikov function $M_{2}$ will be needed.

We compute first the tangent space to $\mathcal{U}_{n / k}$ at a general point $(a, 0)$. Consider for this purpose the one-parameter deformation

$$
\mathcal{F}_{\varepsilon}: \frac{\mathrm{d} y}{y^{2}}=a(x) \mathrm{d} x-\varepsilon \omega_{1}-\varepsilon \omega_{2}-\ldots
$$

where

$$
\omega_{i}=p_{i}(x) \mathrm{d} x+y q_{i}(x) \mathrm{d} x
$$

are polynomial one-forms, $\operatorname{deg} p_{i} \leqslant n, \operatorname{deg} q_{i} \leqslant n$. As before we denote

$$
A=\int a, \quad P_{i}=\int p_{i}, \quad Q_{i}=\int q_{i}
$$

where

$$
A(x)=\widetilde{A}(W(x)), \quad W(0)=W(1), \quad P_{i}(0)=P_{i}(1), \quad Q_{i}(0)=Q_{i}(1) .
$$

The point $(a, 0)$ belongs to $\mathcal{U}_{n / k}$ if and only if $A=\widetilde{A} \circ W$ for some degree $k$ polynomial $W$. 
Proposition 4.4. - The tangent space $T_{(a, 0)} \mathcal{U}_{n / k}$ is the vector space of polynomials $\left(p_{1}, q_{1}\right)$ such that

$$
P_{1}(x)=\widetilde{P}_{1} \circ W(x)+R(x) \cdot \widetilde{A}^{\prime}(W(x)), \quad Q_{1}(x)=\widetilde{Q}_{1}(W(x))
$$

where $\widetilde{P}_{1}, \widetilde{Q}_{1}$ are arbitrary polynomials of degree at most $(n+1) / k$ and $R=R(x)$ is any degree $k$ polynomial, such that $R(0)=R(1)$.

The proof is straightforward, it suffices to consider the first order approximation in $\varepsilon$ of the general deformation

$$
\omega_{1}^{\varepsilon}=\mathrm{d}[(\widetilde{A}+\varepsilon \widetilde{P}) \circ(W+\varepsilon R)(x)]+\varepsilon y \mathrm{~d}[\widetilde{Q} \circ(W+\varepsilon R)(x)]
$$

of $\omega_{1}^{0}=a \mathrm{~d} x$.

Next, we study the tangent cone $T C_{(a, 0)} \mathcal{C}_{n}$. We need to compare the affine varieties $T_{(a, 0)} \mathcal{U}_{n / k} \subset T C_{(a, 0)} \mathcal{C}_{n}$

Proposition 4.5. - In a sufficiently small neighbourhood of every general point $(p, q) \in T_{(a, 0)} \mathcal{U}_{n / k}$ the tangent cones $T C_{(a, 0)} \mathcal{C}_{n}$ and $T_{(a, 0)} \mathcal{U}_{n / k}$ coincide.

The above Proposition 4.5 shows that there is no irreducible component of $T C_{(a, 0)} \mathcal{C}_{n}$ which contains an irreducible component of $T_{(a, 0)} \mathcal{U}_{n / k}$ of strictly smaller dimension. This would imply Theorem 4.1.

The first Melnikov function, as in the case $k=n$, is $M_{1}=\int_{0}^{1} p_{1} \mathrm{~d} x+y q_{1} \mathrm{~d} y$. By Christopher's theorem $M_{1}=0$ implies that $q_{1}$ satisfies the composition condition

$$
Q_{1}(x)=\widetilde{Q}_{1}(W(x)) .
$$

Additional obstructions on the form of $p_{1}$ will be found by inspecting the second Melnikov function $M_{2}$. Under the condition that $M_{1}=0$ we find [Gav05, Formula (2.8)]

$$
M_{2}=\int_{0}^{1} \omega_{1} \omega_{1}^{\prime}+\int_{0}^{1} \omega_{2}
$$

where the derivative' is with respect to the parameter $h$. The identity $h=A(x)+\frac{1}{y}$ shows that $y^{\prime}=-y^{2}$ and $\omega_{1}^{\prime}=-y^{2} \mathrm{~d} x$, it is clearly a covariant derivative in a cohomology bundle (although we do not need this interpretation here). Therefore, for the iterated integral of length two we find

$$
\begin{aligned}
\int_{0}^{1} \omega \omega^{\prime} & =-\int_{\{H=h\}}\left(p_{1} \mathrm{~d} x+q_{1} y \mathrm{~d} x\right)\left(y^{2} q_{1} \mathrm{~d} x\right) \\
& =-\int_{\{H=h\}}\left(p_{1} \mathrm{~d} x\right)\left(y^{2} q_{1} \mathrm{~d} x\right) \\
& =\int_{\{H=h\}} y^{2} q_{1} P_{1} \mathrm{~d} x
\end{aligned}
$$

where $P_{1}$ is a primitive of $p_{1}$. Indeed, $M_{1}=0$ implies the composition condition for $Q_{1}=\int q_{1}$ and $A$, that is to say the integral $\int_{\{H=h\}} y q_{1} \mathrm{~d} x$ vanishes as a pull back. The same then holds true for its derivative $\int_{\{H=h\}} y^{2} q_{1} \mathrm{~d} x$ as well for the iterated integral $\left.\int_{\{H=h\}}\left(y q_{1} \mathrm{~d} x\right)\right)\left(y^{2} q_{1} \mathrm{~d} x\right)$. Further, by the shuffle relation for iterated integrals

$$
\int_{\{H=h\}}\left(p_{1} \mathrm{~d} x\right)\left(y^{2} q_{1} \mathrm{~d} x\right)+\int_{\{H=h\}}\left(y^{2} q_{1} \mathrm{~d} x\right)\left(p_{1} \mathrm{~d} x\right)=\int_{\{H=h\}} p_{1} \mathrm{~d} x \int_{\{H=h\}} y^{2} q_{1} \mathrm{~d} x=0 .
$$


Further, for $\int_{0}^{1} \omega_{2}$ we find

$$
\begin{aligned}
\int_{0}^{1} \omega_{2}=\int_{0}^{1}\left(p_{2}+y q_{2}\right) \mathrm{d} x & =\int_{0}^{1} \frac{\mathrm{d} Q_{2}}{h-A(x)} \\
& =-\int_{0}^{1} \frac{Q_{2} d A}{(h-A)^{2}}+\left.\frac{Q_{2}}{h-A}\right|_{0} ^{1} \\
& =-\int_{0}^{1} y^{2} Q_{2} a \mathrm{~d} x,
\end{aligned}
$$

so that under the condition $M_{1}=0$ implies

$$
M_{2}(h)=\int_{\{H=h\}} y^{2} q_{1} P_{1} \mathrm{~d} x-y^{2} Q_{2} a \mathrm{~d} x=\int_{0}^{1} \frac{q_{1}(x) P_{1}(x)-Q_{2}(x) a(x)}{(h-A(x))^{2}} \mathrm{~d} x .
$$

We apply Christopher's theorem to $M_{2}$ and conclude that the primitive of the polynomial $q_{1}(x) P_{1}(x)-Q_{2}(x) a(x)$ is a composite polynomial, it can be expressed as a polynomial function in $W(x)$, and therefore

$$
q_{1}(x) P_{1}(x)-Q_{2}(x) a(x)=P(W(x)) W^{\prime}(x)
$$

or equivalently

$$
\widetilde{Q}_{1}^{\prime}(W(x)) P_{1}(x)-Q_{2}(x) \widetilde{A}^{\prime}(W(x))=R_{1}(W(x))
$$

for certain polynomial $R_{1}$. Assuming that $\widetilde{Q}_{1}^{\prime}$ and $\widetilde{A}^{\prime}$ are mutually prime, there exist polynomials $R_{2}, R_{3}$ such that

$$
\widetilde{Q}_{1}^{\prime}(W) R_{2}(W)-\widetilde{A}^{\prime}(W) R_{3}(W)=R_{1}(W)
$$

SO

$$
\widetilde{Q}_{1}^{\prime}(W(x))\left(P_{1}(x)-R_{2}(W(x))\right)-\left(Q_{2}(x)-R_{3}(W(x))\right) \widetilde{A}^{\prime}(W(x))=0 .
$$

This implies finally that $\widetilde{A}^{\prime}(W(x))$ divides $P_{1}(x)-R_{2}(W(x))$ and

$$
P_{1}(x)=R_{2}(W(x))+R(x) \widetilde{A}^{\prime}(W(x)) .
$$

Proposition 4.4, and hence Theorem 4.1 is proved.

\subsection{The center set of plane quadratic vector fields}

Let $\mathcal{A}_{n}$ be here the set of all polynomial vector fields of degree at most $n$. The only (non-trivial) case in which the center set $\mathcal{C}_{n} \subset \mathcal{A}_{n}$ is completely known is the quadratic one, $n=2$. For comprehensive description and historical comments concerning the center-focus problem in the quadratic case see Zoladek [Żoł94]. To the plane quadratic vector field (1.1) we associate a foliation $\mathcal{F}_{\omega}=\{\omega=0\}$ on $\mathbb{C}^{2}$, defined by the polynomial one-form

$$
\omega=P(x, y) \mathrm{d} y-Q(x, y) \mathrm{d} x .
$$

The leaves of the foliation are the orbits of the plane vector field (1.1), and the restriction of the one-form $\omega$ on the leaves of $\mathcal{F}_{\omega}$ vanishes identically.

In this section we assume that the polynomials $P, Q$ are of degree at most two, and the system has a center. As the foliation is over $\mathbb{C}$ we must be more careful 
in the definition. We shall say that a singular point is a center, if the point is nondegenerate, and has a local holomorphic first integral with a Morse critical point. Thus, in a neighbourhood of such a point, and up to a complex affine change of the variables, the system can be written in the form

$$
\dot{x}=x+P_{2}(x, y), \quad \dot{y}=-y+Q_{2}(x, y)
$$

for some homogeneous polynomials $P_{2}, Q_{2}$. The following classical result is implicit in Zoladek [Żoł94, Theorem 1]

TheOREM 4.6. - The center set $\mathcal{C}_{2}$ of plane polynomial quadratic systems with a Morse center has four irreducible components.

The above claim is a modern interpretation of the Dulac's classification [Dul08] of such Morse centers in a complex domain, see Lins Neto [LN14, Theorem 1.1]. Indeed, it is easier to decide that a given variety is irreducible, than to decide that it is an irreducible component of some algebraic set. Sketch of the proof of Theorem 4.6 can be found in [FGX16, Appendix].

To describe explicitly the four components of the center variety $\mathcal{C}_{2}$, recall that the foliation $\mathcal{F}_{\omega}$, respectively the vector field (1.1), is said to be logarithmic, if

$$
P(x, y) \mathrm{d} y-Q(x, y) \mathrm{d} x=f_{1} \ldots f_{k} \sum_{i=1}^{k} \lambda_{i} \frac{\mathrm{d} f_{i}}{f_{i}}, \quad f_{i} \in K[x, y], \quad \lambda_{i} \in K
$$

for suitable polynomials $f_{i}$ and exponents $\lambda_{i}$. As

$$
\sum_{i=1}^{k} \lambda_{i} \frac{\mathrm{d} f_{i}}{f_{i}}=\mathrm{d} \log \prod_{i=1}^{k} f_{i}^{\lambda_{i}}
$$

then the logarithmic foliation $\mathcal{F}_{\omega}$ has a first integral of Darboux type

$$
\prod_{i=1}^{k} f_{i}^{\lambda_{i}} .
$$

Let $\mathcal{L}\left(d_{1}, d_{2}, \ldots, d_{k}\right)$ denotes the set of such logarithmic foliations (or plane vector fields) with

$$
\operatorname{deg} f_{1} \leqslant d_{1}, \operatorname{deg} f_{2} \leqslant d_{2}, \ldots, \operatorname{deg} f_{k} \leqslant d_{k} .
$$

For generic polynomials $f_{i}$ of degree $d_{i}$ the degree of the associated vector field is $\sum d_{i}-1$. Therefore $\mathcal{L}\left(d_{1}, d_{2}, \ldots, d_{k}\right)$ is quadratic, provided that $d_{1}=3$ or $d_{1}=1$, $d_{2}=2$ or $d_{1}=d_{2}=d_{3}=1$. This defines three large irreducible components of the center set $\mathcal{C}_{2}$ of quadratic systems with a Morse center, $\mathcal{L}(3), \mathcal{L}(1,2), \mathcal{L}(1,1)$ respectively. We have, however, one more irreducible component of $\mathcal{C}_{2}$ which is

$$
\mathcal{Q}_{4}=\mathcal{L}(2,3) \cup \mathcal{A}_{2} .
$$

Here $\mathcal{L}(2,3)$ is the set of polynomial foliations as above, with a first integral $f_{2}^{3} / f_{3}^{2}$ where $\operatorname{deg} f_{2}=2 \operatorname{deg} f_{3}=3$. Generically such a foliation is of degree four, but it happens that its intersection $\mathcal{Q}_{4}$ with the space $\mathcal{A}_{2}$ of quadratic foliations is non empty and it is an irreducible algebraic set. The notation $\mathcal{Q}_{4}$ is introduced by Zoladek [Żoł94], the index 4 indicates the co-dimension of the set in the space of quadratic vector fields $\mathcal{A}_{2}$. 
The exceptional set $\mathcal{Q}_{4}$ might look not quite explicit, we investigate it in details below.

The space $\mathcal{A}_{n}$ of polynomial vector fields of degree at most $n$ are identified to a vector space of dimension $(n+1)(n+2)$. On $\mathcal{A}_{n}$ acts the affine group Aff $_{2}$ of affine transformations of $K^{2}$ (as usual $K=\mathbb{R}$ or $K=\mathbb{C}$ ), as well the multiplicative group $K^{*}$ corresponding to "change of time", $\operatorname{dim} A f f_{2} \times K^{*}=7$. Therefore the dimension of the orbit of a general polynomial vector field is 7 . For this reason it is expected that the minimal dimension of a component of the center set $\mathcal{C}_{n}$ is also 7 . Such components, if exist, will be in a sense exceptional.

In the quadratic case $n=2$ the dimension of the four components of $\mathcal{C}_{2}$ are easily found. For instance, in the case $\mathcal{L}(1,1,1) \subset \mathcal{A}_{2}$, and up to an affine changes of variables and time, one may suppose that the first integral is in the form $x y^{\lambda}(1-$ $x-y)^{\mu}$. Therefore the dimension of $\mathcal{L}(1,1,1)$ is $2+7=9$ and the codimension is $3=12-9$. We find similarly that $\operatorname{dim} \mathcal{L}(2,1)=\operatorname{dim} \mathcal{L}(3)=9$.

We describe now the last component $\mathcal{Q}_{4}$. Let $[x: y: z]$ be homogeneous coordinates in $\mathbb{P}^{2}$

$$
\begin{aligned}
& P_{2}(x, y, z)=a_{2}(x, y)+a_{1}(x, y) z+a_{0}(x, y) z^{2} \\
& P_{3}(x, y, z)=b_{3}(x, y)+b_{2}(x, y) z+b_{1}(x, y) z^{2}+b_{0}(x, y) z^{3}
\end{aligned}
$$

be homogeneous polynomials in $x, y, z$ of degree 2 and 3 . The function

$$
H=P_{2}^{3} / P_{3}^{2}
$$

is therefore rational on $\mathbb{P}^{2}$ and induces a foliation on $\mathbb{P}^{2}$

$$
3 P_{3}(x, y, z) \mathrm{d} P_{2}(x, y, z)-2 P_{2}(x, y, z) \mathrm{d} P_{3}(x, y, z)=0 .
$$

The corresponding affine foliation on the chart $\mathbb{C}^{2}$ defined by $z=1$

$$
3 P_{3}(x, y, 1) \mathrm{d} P_{2}(x, y, 1)-2 P_{2}(x, y, 1) \mathrm{d} P_{3}(x, y, 1)=0
$$

is of degree 4 . We may obtain a plane polynomial foliation of degree 2 by imposing the following additional conditions.

Suppose first, that the infinite line $\{z=0\}$ is invariant, that is to say (up to affine change)

$$
H(x: y: 1)=\frac{a_{2}(x, y)^{3}}{b_{3}(x, y)^{2}}=1 .
$$

This condition can be written as

$$
P_{2}(x, y, z)^{3}=P_{3}(x, y, z)^{2}+O(z) .
$$

The foliation (4.9) takes the form

$$
z[P(x, y, z) \mathrm{d} x+Q(x, y, z) \mathrm{d} y]+R(x, y, z) \mathrm{d} z=0
$$

where $\operatorname{deg} P, \operatorname{deg} Q \leqslant 3$, so (4.10) is of degree 3 . If we further suppose that $z$ divides the homogeneous one form $3 P_{3} \mathrm{~d} P_{2}-2 P_{2} \mathrm{~d} P_{3}$ then (4.9) takes the form

$$
z^{2}[P(x, y, z) \mathrm{d} x+Q(x, y, z) \mathrm{d} y]+z R(x, y, z) \mathrm{d} z=0
$$


where $\operatorname{deg} P, \operatorname{deg} Q \leqslant 2$, so (4.10) is a plane quadratic foliation. The condition that $z^{2}$ divides $2 P_{3} \mathrm{~d} P_{2}-3 P_{2} \mathrm{~d} P_{3}$ can be written as

$$
P_{2}(x, y, z)^{3}=P_{3}(x, y, z)^{2}+O\left(z^{2}\right)
$$

or equivalently

$$
\begin{aligned}
a_{2}(x, y)^{3} & =b_{3}(x, y)^{2} \\
3 a_{2}(x, y)^{2} a_{1}(x, y) & =2 b_{3}(x, y) b_{2}(x, y) .
\end{aligned}
$$

These polynomial relations can be further simplified by affine changes of the variables $x, y$. First, (4.12) implies that $a_{2}$ is a square of a linear function in $x, y$ which we may suppose equal to $x$, that is to say

$$
a_{2}(x, y)=x^{2}, b_{3}(x, y)=x^{3} .
$$

The second condition (4.13) becomes $3 x a_{1}=2 b_{2}$ where we may put $a_{1}=2 y$, and hence

$$
a_{1}(x, y)=2 y, b_{2}(x, y)=3 x y .
$$

It is seen that the polynomial $P_{3}(x, y, 1)$ has a real critical point which we can put at the origin, so we shall also suppose that $b_{1}=0$. Using finally a "change of time" (the action of $K^{*}$ ) we assume that $b_{0}=1$ while $a_{0}=\alpha \in K$ is a free parameter (modulus). The first integral takes therefore the form

$$
H_{\alpha}(x, y)=\frac{\left(x^{2}+2 y+\alpha\right)^{3}}{\left(x^{3}+3 x y+1\right)^{2}}
$$

with induced quadratic foliation

$$
\left(-\alpha x^{2}-2 y^{2}-\alpha y+x\right) \mathrm{d} x+(x y-\alpha x+1) \mathrm{d} y .
$$

This is the exceptional co-dimension four component of $Q_{4}$.

The reader may check that the corresponding vector field

$$
x^{\prime}=x y-\alpha x+1, \quad y^{\prime}=\alpha x^{2}+2 y^{2}+\alpha y-x
$$

has a Morse center at $x=1 / \alpha, y=0$ which is moreover a usual real center for $\alpha \in$ $(1,0)$. The above computation is suggested by [LN14] where, however, the modulus $\alpha$ is wrongly fixed equal to $\alpha=\infty$ ). The foliation on $\mathbb{P}^{2}$ corresponding to

$$
H_{\infty}(x, y)=\frac{\left(x^{2}+2 y+1\right)^{3}}{\left(x^{3}+3 x y\right)^{2}}
$$

has two invariant lines $\{x=0\}$ and $\{z=0\}$, in contrast to the general foliation defined by $d H_{\alpha}(x, y)=0$ which has only one invariant line $\{z=0\}$. We resume the above as follows

Proposition 4.7. - Every polynomial vector field having a rational first integral of the form

$$
H(x, y)=\frac{\left(a_{0}(x, y)+a_{1}(x, y)+a_{2}(x, y)\right)^{3}}{\left(b_{0}(x, y)+b_{1}(x, y)+b_{2}(x, y)+b_{3}(x, y)\right)^{2}}
$$


where the homogeneous polynomials $a_{i}, b_{j}$ of degrees $0 \leqslant i \leqslant 2,0 \leqslant j \leqslant 3$ are subject to the relations

$$
\begin{aligned}
a_{2}(x, y)^{3} & =b_{3}(x, y)^{2} \\
3 a_{2}(x, y)^{2} a_{1}(x, y) & =2 b_{3}(x, y) b_{2}(x, y)
\end{aligned}
$$

is of degree two. The set of such quadratic vector fields form the irreducible component $\mathcal{Q}_{4}$ of the center set $\mathcal{C}_{2}$. Up to an affine change of the variables $x, y$ the polynomial $H$ can be assumed in the form $H(x, y)=\frac{\left(x^{2}+2 y+\alpha\right)^{3}}{\left(x^{3}+3 x y+1\right)^{2}}$ where $\alpha$ is a parameter.

We conclude this section with the following remarkable property of $\mathcal{Q}_{4}$. One may check that general rational function of the form $H(x, y)=P_{2}^{3} / P_{3}^{2}$, where $P_{2}, P_{3}$ are bi-variate polynomials of degree two and three, defines a pencil of genus four curves $\Gamma_{t}=\{(x, y): H(x, y)=t\}$ on $\mathbb{C}^{2}$. However, the special rational function $H_{\alpha}(4.14)$ defines an elliptic pencil, that is to say the level sets

$$
\Gamma_{t}=\left\{(x, y) \in \mathbb{C}^{2}: H_{\alpha}(x, y)=t\right\}
$$

are genus one curves, see [GI09].

\subsection{The center set of the polynomial Liénard equation}

Consider the following polynomial Liénard equation

$$
\dot{x}=y, \quad \dot{y}=-q(x)-y p(x)
$$

in which the origin $(0,0)$ is an isolated singular point. The following is an obvious necessary and sufficient condition the equation (4.16) to have a linear center

$$
q(0)=p(0)=0, \quad q^{\prime}(0)>0 .
$$

The description of the non-degenerate centers of (4.16) is due to Cherkas [Che72] in the real analytic case, and to Christopher [Chr99] in the polynomial case.

Consider the following Polynomial Composition Condition (PCC)

There exist polynomials $\widetilde{P}, \widetilde{Q}, W$ such that

$$
P=\widetilde{P} \circ W, \quad Q=\widetilde{Q} \circ W
$$

where $P^{\prime}(x)=p(x), Q^{\prime}(x)=q(x)$.

The Theorem of Cherkas and Christopher can be formulated as follows

THEOREM 4.8. - The real polynomial Liénard equation (4.16) has a non-degenerate real center at the origin in $\mathbb{R}^{2}$, if and only if

$$
p(0)=q(0)=0, \quad q^{\prime}(0)>0
$$

and the polynomials $p, q$ satisfy the above Polynomial Composition Condition, where the real polynomial $W$ has a Morse critical point at the origin.

The proof of Theorem 4.8, see [Chr99, CL07], is based on the following simple observation due to Cherkas [Che72, Lemma 1] 
Lemma 4.9. - The real analytic equation

$$
\dot{x}=y, \quad \dot{y}=-x+y \sum_{i=1}^{\infty} a_{i} x^{i}
$$

has a center at the origin, if and only if $a_{2 j}=0, \forall j \geqslant 1$.

Indeed, the truncated equation

$$
\dot{x}=y, \quad \dot{y}=-x+y \sum_{j=0}^{\infty} a_{2 j+1} x^{2 j+1}
$$

is reversible in $x$, and hence it has a center at the origin. In a sufficiently small neighbourhood of the origin, (4.18) is rotated with respect to the vector field (4.17), unless $a_{2 j}=0, \forall j \geqslant 1$. The final argument of Christopher is to use the Lüroth theorem, to deduce the (PCC) condition. This topological argument of Cherkas does not apply in a complex domain. We shall prove, however, the following more general

Theorem 4.10. - The (possibly complex) polynomial Liénard equation (4.16) has a Morse critical point at the origin in $\mathbb{C}^{2}$, if and only if

$$
p(0)=q(0)=0, \quad q^{\prime}(0) \neq 0
$$

and the polynomials $p, q$ satisfy the above Polynomial Composition Condition, where the (possibly complex) polynomial $W$ has a Morse critical point at the origin.

Theorem 4.10 implies Theorem 4.8. Its meaning is, that the origin is a Morse center if and only if the Liénard equation is a "pull back". More precisely, the Liénard equation (4.16) induces a polynomial foliation

$$
y \mathrm{~d} y+(q(x)+y p(x)) \mathrm{d} x=0 .
$$

which is pull back of the foliation

$$
y \mathrm{~d} y+\mathrm{d} \widetilde{Q}(W)+y \mathrm{~d} \widetilde{P}(W)=0
$$

under the map $W=W(x), y=y$. We may assume that $\widetilde{Q}(0)=\widetilde{P}(0)=0$ and $\widetilde{Q}^{\prime}(0) \neq 0$. Under these hypothesis $(4.20)$ has a local first integral of the form

$$
W+c_{1} y^{2}+c_{2} W y+c_{2} W^{2}+\ldots, \quad c_{1} \neq 0
$$

which implies that the pull back foliation (4.19) has an analytic first integral with a Morse critical point at the origin.

To the end of this subsection 4.3 we prove Theorem 4.10. Assume that the Liénard equation (4.16) has a Morse critical point at the origin. As $q(0)=0, q^{\prime}(0) \neq 0$ and $p(0)=0$, then the polynomial $\frac{1}{2} y^{2}+Q(x)$ has a Morse critical point at the origin and there exists a local bi-analytic change of the variable $x \rightarrow X$ such that $\frac{1}{2} y^{2}+Q(x(X))=\frac{1}{2}\left(y^{2}+X^{2}\right)$. Thus

$$
y \mathrm{~d} y+(q(x)+y p(x)) \mathrm{d} x=\frac{1}{2} \mathrm{~d}\left(y^{2}+X^{2}\right)+y \mathrm{~d} P(x(X)) .
$$

We expand

$$
\mathrm{d} P(x(X))=\left(\sum_{i=1}^{\infty} a_{i} X^{i}\right) \mathrm{d} X
$$


and obtain the equivalent foliation

$$
\frac{1}{2} \mathrm{~d}\left(y^{2}+X^{2}\right)+y\left(\sum_{i=1}^{\infty} a_{i} X^{i}\right) \mathrm{d} X=0 .
$$

By analogy to the Cherkas Lemma we shall prove

Lemma 4.11. - The foliation (4.22) has a Morse critical point at the origin if and only if $a_{2 j}=0, \forall j \geqslant 1$.

Proof. - After rescaling $(X, y) \mapsto \varepsilon(X, y)$ the foliation takes the form

$$
\mathcal{F}_{\varepsilon}: \frac{1}{2} \mathrm{~d}\left(y^{2}+X^{2}\right)+y\left(\sum_{i=1}^{\infty} \varepsilon^{i} a_{i} X^{i}\right) \mathrm{d} X=0
$$

and it suffices to prove that for sufficiently small $\varepsilon$ it has a Morse critical point. Note first that the truncated foliation $\mathcal{F}_{\varepsilon}^{t}$

$$
\mathcal{F}_{\varepsilon}^{t}: \frac{1}{2} \mathrm{~d}\left(y^{2}+X^{2}\right)+y\left(\sum_{j=0}^{\infty} \varepsilon^{2 j+1} a_{2 j+1} X^{2 j+1}\right) \mathrm{d} X=0
$$

is a pullback of

$$
\frac{1}{2} \mathrm{~d}\left(y^{2}+\xi\right)+\frac{1}{2} y\left(\sum_{j=0}^{\infty} \varepsilon^{2 j+1} a_{2 j+1} \xi^{j}\right) \mathrm{d} \xi=0
$$

under the map $\pi:(X, y) \mapsto(\xi, y), \xi=X^{2}$. The foliation (4.25) is regular at the origin and has a first integral

$$
\frac{1}{2}\left(y^{2}+\xi\right)+O(\varepsilon)
$$

where $O(\varepsilon)$ is analytic in $\varepsilon, \xi, Y$, and vanishes as $\varepsilon=0$. Thus $\mathcal{F}_{\varepsilon}^{t}$ has a first integral

$$
H_{\varepsilon}(x, y)=\frac{1}{2}\left(y^{2}+X^{2}\right)+O(\varepsilon)
$$

where $O(\varepsilon)$ is analytic in $\varepsilon, X^{2}, Y$, and vanishes as $\varepsilon=0$. This also shows that the origin is a Morse critical point of the truncated foliation $\mathcal{F}_{\varepsilon}^{t}$.

As $H_{\varepsilon}$ is a first integral of $\mathcal{F}_{\varepsilon}^{t}$ then for every fixed $\varepsilon$ we have

$$
(1+O(\varepsilon)) \mathrm{d} H_{\varepsilon}(x, y)=\frac{1}{2} \mathrm{~d}\left(y^{2}+X^{2}\right)+y\left(\sum_{j=0}^{\infty} \varepsilon^{2 j+1} a_{2 j+1} X^{2 j+1}\right) \mathrm{d} X .
$$

Suppose now that for some $j \geqslant 1, a_{2 j} \neq 0$ and let $j=k$ be the smallest integer with this property. We have

$$
\mathcal{F}_{\varepsilon}:(1+O(\varepsilon)) \mathrm{d} H_{\varepsilon}(x, y)+\varepsilon^{2 k} y a_{2 k} X^{2 k} \mathrm{~d} X+O\left(\varepsilon^{2 k+1}\right) \mathrm{d} X=0
$$

where by abuse of notations $O\left(\varepsilon^{2 k+1}\right)$ denotes an analytic function in $X, y, \varepsilon$ which is divisible by $\varepsilon^{2 k+1}$. The origin is a Morse critical point if and only if the holonomy map of the two separatrices of $\mathcal{F}_{\varepsilon}$ at the origin, are the identity maps. The holonomy map will be evaluated by the usual Poincaré-Pontryagin-Melnikov formula. The 
separatrices are tangent to the lines $y \pm i X=0$. We take a cross-section to one of the separatrices, parameterised by the restriction of $H_{\varepsilon}(x, y)$ on it. Let

$$
\gamma_{\varepsilon}(h) \subset\left\{(x, y): H_{\varepsilon}(x, y)=h\right\}
$$

be a continuous family of closed loops vanishing at the origin as $h \rightarrow 0$. The holonomy map of $\mathcal{F}_{\varepsilon}$, corresponding to this closed loop is

$$
\begin{aligned}
h \mapsto h+\frac{\varepsilon^{2 k}}{1+O(\varepsilon)}\left(\int_{\gamma_{\varepsilon}(h)} y a_{2 k} X^{2 k} \mathrm{~d} X\right. & +O(\varepsilon) \mathrm{d} X) \\
& =h+\varepsilon^{2 k} \int_{\gamma_{\varepsilon}(h)} y a_{2 k} X^{2 k} \mathrm{~d} X+O\left(\varepsilon^{2 k+1}\right) \mathrm{d} X \\
& =h+\varepsilon^{2 k} a_{2 k} \int_{\gamma_{0}(h)} y X^{2 k} \mathrm{~d} X+O\left(\varepsilon^{2 k+1}\right) \mathrm{d} X
\end{aligned}
$$

where

$$
\gamma_{0}(h) \subset\left\{(x, y): H_{0}(x, y)=h\right\}=\left\{(x, y): \frac{1}{2}\left(y^{2}+X^{2}\right)=h\right\} .
$$

By homogeneity of the polynomials

$$
\int_{\gamma_{0}(h)} y X^{2 k} \mathrm{~d} X=h^{k+1} \int_{\gamma_{0}(1)} y X^{2 k} \mathrm{~d} X .
$$

As the homology of the algebraic curve $\left\{(y, X) \in \mathbb{C}^{2}: y^{2}+X^{2}=2 h\right\}$ has one generator we can suppose that this generator is just the real circle $\gamma_{0}(1)=\{(y, X) \in$ $\left.\mathbb{R}^{2}: y^{2}+X^{2}=2\right\}$ and in this case

$$
\int_{\gamma_{0}(1)} y X^{2 k} \mathrm{~d} X=\iint_{y^{2}+X^{2} \leqslant 2} X^{2 k} \mathrm{~d} X \mathrm{~d} y \neq 0
$$

We conclude that if the holonomy map is the identity map, then $a_{2 k}=0$ which is the desirable contradiction. Lemma 4.11 is proved.

Proof of Theorem 4.10. - Assuming that the Liénard equation has a Morse critical point, and hence $Q(x)$ has a Morse critical point at the origin, denote $x_{1}(h), x_{2}(h)$ the two roots of the polynomial $Q(x)-h$ which vanish at 0 as $h$ tends to 0 . We have obviously that $X\left(x_{1}(h)\right)=-X\left(x_{2}(h)\right)$. By Lemma 4.11 the analytic function $P(x(X))$ is even in $X$, and hence $P\left(x_{1}(h)\right) \equiv P\left(x_{2}(h)\right)$. Following an idea of Christopher (already used at the end of Section 3), consider now the subfield $\mathcal{C} \subset \mathbb{C}(x)$ formed by all rational functions $R=R(x) \in \mathbb{C}(x)$ satisfying the identity

$$
R\left(x_{1}(h)\right) \equiv R\left(x_{2}(h)\right) .
$$

According to the Lüroth theorem, every subfield of $\mathbb{C}(x)$ is of the form $\mathbb{C}(W)$ for some rational function $W=W(x)$. Thus we have $\mathcal{C}=\mathbb{C}(W)$ where $P, Q \in \mathcal{C}$. Therefore there exist rational functions $\widetilde{P}, \widetilde{Q}$ such that

$$
P=\widetilde{P} \circ W, \quad Q=\widetilde{Q} \circ W .
$$

Using the same argument as in the proof of Theorem 3.2 we may suppose that $\widetilde{P}, W, \widetilde{Q}$ are polynomials, and hence $P, Q$ satisfy (PCC) which completes the proof of the Theorem 4.10. 


\subsection{Abel equations with Darboux type first integral}

The polynomial Liénard equation

$$
\dot{x}=y, \quad \dot{y}=-q(x)-y p(x)
$$

with associated foliation $y \mathrm{~d} y+(q(x)+y p(x)) \mathrm{d} x=0$, after the substitution $y \rightarrow 1 / y$, becomes the following Abel equation

$$
\frac{\mathrm{d} y}{\mathrm{~d} x}=y^{2} p(x)+y^{3} q(x) .
$$

Equivalently, we consider the foliation

$$
\mathrm{d} y=\left(y^{2} p(x)+y^{3} q(x)\right) \mathrm{d} x .
$$

The classification of Morse critical points of the Liénard equation (4.16) obtained in Section 4.3 suggests that a similar claim would hold true for the scalar Abel equation (4.29). This is the content of the following

Composition Conjecture [BRY10, p. 444]. - The Abel equation (4.29) has a center at the solution $y=0$ along some fixed interval $[a, b]$ if and only if the following Polynomial Composition Condition (PCC) holds true

$$
P=\widetilde{P} \circ W, Q=\widetilde{Q} \circ W, W(a)=W(b) .
$$

Note that the Cherkas-Christopher theorem is for non-degenerate centers. The Composition Conjecture missed the possibility for the Abel or Liénard equations to have a Darboux type first integral, with resonant saddle point and characteristic ratio $p:-q$ (instead of a non-degenerate center with $1:-1$ ratio). Incidentally, Liénard equations with a Darboux type first integral will produce counter-examples to the Composition Conjecture, which is the subject of the present section. We explain in this context the recent counter-example of Giné, Grau and Santallusia [GGS19].

The method of constructing such systems is based on the example of the co-dimension four center set $\mathcal{Q}_{4}$ for quadratic system, as explained in Section 4.2.

Let

$$
\begin{aligned}
& P_{2}=a_{0}(x)+a_{1}(x) y+a_{2}(x) y^{2} \\
& Q_{2}=b_{0}(x)+b_{1}(x) y+b_{2}(x) y^{2}
\end{aligned}
$$

where $a_{i}, b_{j}$ are polynomials, such that $P_{2}^{p}=Q_{2}^{q}+O\left(y^{3}\right)$, where $p, q$ are positive relatively prime integers. This implies that the corresponding one-form

$$
p Q_{2} \mathrm{~d} P_{2}-q P_{2} \mathrm{~d} Q_{2}
$$

is divisible by $y^{2}$, and then the associated reduced foliation (after division by $y^{2}$ ) is of degree two in $y$, and moreover $\{y=0\}$ is a leaf. Therefore the foliation is defined as

$$
\left(r_{1} y+r_{2}\right) \mathrm{d} y=y\left(r_{3} y+r_{4}\right) \mathrm{d} x=0, \quad r_{i} \in \mathbb{C}[x]
$$


where

$$
\begin{aligned}
& r_{1}=2(p-q) a_{2} b_{2} \\
& r_{2}=(p-2 q) a_{1} b_{2}-(q-2 p) b_{1} a_{2} \\
& r_{3}=p a_{2}^{\prime} b_{2}-q b_{2}^{\prime} a_{2} \\
& r_{4}=p a_{1}^{\prime} b_{2}-q b_{1}^{\prime} a_{2} .
\end{aligned}
$$

Note that if $a_{2}=$ const. $\neq 0, b_{2}=$ const. $\neq 0$ the foliation takes the Liénard form

$$
\left(r_{1} y+r_{2}\right) \mathrm{d} y=y r_{4} \mathrm{~d} x, \quad r_{1}=\text { const. . }
$$

Of course, it is not clear, whether such polynomials exist. To verify this we have to solve the equation

$$
\left(a_{0}(x)+a_{1}(x) y+a_{2}(x) y^{2}\right)^{p}=\left(b_{0}(x)+b_{1}(x) y+b_{2}(x) y^{2}\right)^{q} \bmod y^{3}
$$

assuming that $a_{i}(x), b_{j}(x)$ are polynomials, and $a_{2}, b_{2}$ are constants. A first condition is given by

which implies

$$
a_{0}^{p}=b_{0}^{q}
$$

$$
\left(1+\frac{a_{1}(x)}{a_{0}(x)} y+\frac{a_{2}(x)}{a_{0}(x)} y^{2}\right)^{p}=\left(1+\frac{b_{1}(x)}{b_{0}(x)} y+\frac{b_{2}(x)}{b_{0}(x)} y^{2}\right)^{q} \bmod y^{3}
$$

or equivalently

$$
\begin{aligned}
p \frac{a_{1}(x)}{a_{0}(x)} & =q \frac{b_{1}(x)}{b_{0}(x)} \\
p \frac{a_{2}(x)}{a_{0}(x)}+\frac{p(p-1)}{2}\left(\frac{a_{1}(x)}{a_{0}(x)}\right)^{2} & =q \frac{b_{2}(x)}{b_{0}(x)}+\frac{q(q-1)}{2}\left(\frac{b_{1}(x)}{b_{0}(x)}\right)^{2} .
\end{aligned}
$$

Thus $a_{i}, b_{j}$ are polynomials which satisfy the following redundant system of equations

$$
\begin{aligned}
a_{0}(x)^{p} & =b_{0}(x)^{q} \\
p \frac{a_{1}(x)}{a_{0}(x)} & =q \frac{b_{1}(x)}{b_{0}(x)} \\
p \frac{a_{2}(x)}{a_{0}(x)}-\frac{p}{2}\left(\frac{a_{1}(x)}{a_{0}(x)}\right)^{2} & =q \frac{b_{2}(x)}{b_{0}(x)}-\frac{q}{2}\left(\frac{b_{1}(x)}{b_{0}(x)}\right)^{2} .
\end{aligned}
$$

It follows that for some polynomial $R$,

$$
a_{0}(x)=R(x)^{q}, b_{0}=R(x)^{p}
$$

and moreover

$$
p a_{2} R(x)^{-q}-q b_{2} R(x)^{-p}
$$

is a square of a rational function, where we recall that $a_{2}=$ const., $b_{2}=$ const.. It is easy to check that this is only possible if, say $p<q$, and $p=2 k-1, q=2 k$ for an integer $k \geqslant 1$. With this observation the analysis of the system is straightforward and is left to the reader. We formulate the final result in the following 
Theorem 4.12. - For every integer $k \geqslant 1$ and polynomial $r(x)$ the function

$$
H(x, y)=\frac{\left[\left(1-r(x)^{2}\right)^{2 k}+2 k r(x)\left(1-r(x)^{2}\right)^{k} y+k y^{2}\right]^{2 k-1}}{\left[\left(1-r(x)^{2}\right)^{2 k-1}+(2 k-1) r(x)\left(1-r(x)^{2}\right)^{k-1} y+\frac{2 k-1}{2} y^{2}\right]^{2 k}}
$$

is the first integral of a Liénard type equation of the form

$$
\left\{\begin{array}{l}
\frac{\mathrm{d} x}{\mathrm{~d} t}=-y+r_{2}(x), \\
\frac{\mathrm{d} y}{\mathrm{~d} t}=y r_{4}(x)
\end{array}\right.
$$

for suitable polynomials $r_{2}, r_{4}$.

It is clear that the above Liénard system is a polynomial pull back under $x \rightarrow r(x)$ of a simpler master Liénard system with first integral

$$
H_{k}(x, y)=\frac{\left[\left(1-x^{2}\right)^{2 k}+2 k x\left(1-x^{2}\right)^{k} y+k y^{2}\right]^{2 k-1}}{\left[\left(1-x^{2}\right)^{2 k-1}+(2 k-1) x\left(1-x^{2}\right)^{k-1} y+\frac{2 k-1}{2} y^{2}\right]^{2 k}}
$$

which can not be further reduced. As the Liénard equation (4.32) is equivalent to the Abel equation

$$
\frac{\mathrm{d} z}{\mathrm{~d} x}=p(x) z^{2}+q(x) z^{3}
$$

where $z=1 / Y$ and $Y=y-r_{2}(x)$ and

$$
p(x)=r_{4}(x)+r_{2}^{\prime}(x), q(x)=r_{2}(x) r_{4}(x)
$$

then we obtain for every $k \in \mathbb{N}^{*}$ an Abel equation with a Darboux type first integral. Except in the case $k=1$ these Abel equations will have a center along the interval $[-1,1]$.

To the end of the Section 4.4 we consider in more detail the simplest cases $k=1$ and $k=2$. For $k=1$ we get

$$
H_{1}(x, y)=\frac{\left(1-x^{2}\right)^{2}+2 x\left(1-x^{2}\right) y+y^{2}}{\left(1-x^{2}+x y+\frac{1}{2} y^{2}\right)^{2}} .
$$

which is the first integral of the following cubic Liénard equation :

$$
\left\{\begin{array}{l}
\frac{\mathrm{d} x}{\mathrm{~d} t}=y+3 x\left(1-x^{2}\right) \\
\frac{\mathrm{d} y}{\mathrm{~d} t}=-y\left(1+3 x^{2}\right)
\end{array}\right.
$$

The characteristic ratios of the singular points $(0,0),( \pm 1,0)$ are equal to $3:-1$ and $3:-4$ and the characteristic values of $\left(+\frac{1}{\sqrt{3}},-\frac{1}{\sqrt{3}}\right)$ and $\left(-\frac{1}{\sqrt{3}},+\frac{1}{\sqrt{3}}\right)$ equal -1 (so we have integrable cubic saddles, presumably new). The Liénard transformation $Y=2 y+3 x\left(1-x^{2}\right)$ transforms the above to the standard form

$$
\frac{\mathrm{d} x}{\mathrm{~d} t}=Y, \frac{\mathrm{d} Y}{\mathrm{~d} t}=p(x)+q(x) Y \Longleftrightarrow Y \frac{\mathrm{d} Y}{\mathrm{~d} x}=p(x)+q(x) Y
$$

or also to the Abel type equation

$$
\frac{\mathrm{d}}{\mathrm{d} x}(1 / Y)=-q(x)\left(\frac{1}{Y}\right)^{2}-p(x)\left(\frac{1}{Y}\right)^{3}
$$


with respect to the variable $z=1 / Y$.

Assume that $k=2$, the first integrals takes the form

$$
H_{2}(x, y)=\frac{\left(y^{2}-2 x y\left(1-x^{2}\right)^{2}+\frac{1}{2}\left(1-x^{2}\right)^{4}\right)^{3}}{\left(y^{2}-2 x y\left(1-x^{2}\right)+\frac{2}{3}\left(1-x^{2}\right)^{3}\right)^{4}}
$$

while the corresponding foliation of Liénard type is defined by

$$
\left(15 x^{4}-6 x^{2}-1\right) y \mathrm{~d} x-\left(\left(5 x^{2}-3\right)\left(x^{2}-1\right) x+y\right) \mathrm{d} y=0 .
$$

Namely, the Liénard transformation

$$
y \rightarrow-y-\left(5 x^{2}-3\right)\left(x^{2}-1\right) x
$$

transforms the equation (4.36) to

$$
(q(x)+p(x) y) \mathrm{d} x+y \mathrm{~d} y=0
$$

or equivalently to

$$
\left\{\begin{array}{l}
\frac{\mathrm{d} x}{\mathrm{~d} t}=-y \\
\frac{\mathrm{d} y}{\mathrm{~d} t}=q(x)+p(x) y
\end{array}\right.
$$

where

$$
\begin{aligned}
& p(x)=2\left(20 x^{4}-15 x^{2}+1\right) \\
& q(x)=x(x-1)(x+1)\left(5 x^{2}-3\right)\left(15 x^{4}-6 x^{2}-1\right) .
\end{aligned}
$$

The first integral $H_{2}$ takes the form

$$
\frac{\left(y^{2}-8 x\left(1-x^{2}\right)\left(x^{2}-\frac{1}{2}\right) y+\left(1-x^{2}\right)^{2}\left(x^{2}-\frac{1}{2}\right)\left(15 x^{4}-6 x^{2}-1\right)\right)^{3}}{\left(y^{2}-2 x\left(1-x^{2}\right)\left(5 x^{2}-2\right) y+\frac{1}{3}\left(1-x^{2}\right)^{2}\left(5 x^{2}-2\right)\left(15 x^{4}-6 x^{2}-1\right)\right)^{4}} .
$$

However, after the substitution $1 \rightarrow 1 / y$, the above Liénard equation is equivalent to the Abel equation

$$
\frac{\mathrm{d} y}{\mathrm{~d} x}=p(x) y^{2}+q(x) y^{3}
$$

with Darboux type first integral $H(x, y)$ given by the expression

$$
\frac{y^{2}\left[1-8 x\left(1-x^{2}\right)\left(x^{2}-\frac{1}{2}\right) y+\left(1-x^{2}\right)^{2}\left(x^{2}-\frac{1}{2}\right)\left(15 x^{4}-6 x^{2}-1\right) y^{2}\right]^{3}}{\left[1-2 x\left(1-x^{2}\right)\left(5 x^{2}-2\right) y+\frac{1}{3}\left(1-x^{2}\right)^{2}\left(5 x^{2}-2\right)\left(15 x^{4}-6 x^{2}-1\right) y^{2}\right]^{4}} \text {. }
$$

The above first integral of the Abel equation (4.38) has been found first by Giné, Grau and Santallusia [GGS19]. Using this fact they deduced the following

Theorem 4.13. - The Abel equation (4.38) has a center at $y=0$ along the interval $[-1,1]$ but this center is not universal.

Proof. - As $y=0$ is a solution of (4.38) then for all sufficiently small $|\varepsilon|$ the solution $y=y(x)$ with initial condition $y(-1)=\varepsilon \neq 0$ exists along the compact interval $[-1,1]$. The identity $H( \pm 1, y)=y^{2}$ shows that $y(1)=\varepsilon$ or $y(1)=-\varepsilon$ and it is easy to check that in fact $y(1)=\varepsilon$, Indeed, for real $\varepsilon$ the solution $y(x)$ does 
not vanish and hence it has the same sign as $\varepsilon$. Therefore the transport map along the interval $[-1,1]$ is the identity map, and the Abel equation has a center at the solution $y=0$.

The polynomials $P=\int p$ and $Q=\int q$ are of degrees 5 and 10 . Therefore if they had a common non-trivial composition factor, then the factor would be $P$ and $Q=\widetilde{Q} \circ P$ for suitable quadratic polynomial $\widetilde{Q}$. It follows that $p=P^{\prime}$ divides $q=Q^{\prime}$ which is obviously not true. Thus $P, Q$ can not have a common composition factor, and (by the Brudnuy's theorem) the center is not universal.

\section{BIBLIOGRAPHY}

[BFY98] Miriam Briskin, Jean-Pierre Françoise, and Yosef Yomdin, The Bautin ideal of the Abel equation, Nonlinearity 11 (1998), no. 3, 431-443. ^623, 624, 625

[BFY99] Center conditions, compositions of polynomials and moments on algebraic curves, Ergodic Theory Dyn. Syst. 19 (1999), no. 5, 1201-1220. $\uparrow 623,624,625,627$

[BFY00] , Center conditions. II. Parametric and model center problems, Isr. J. Math. 118 (2000), 61-82. $\uparrow 623,624,625$

[Bru06] Alexander Brudnyi, On the center problem for ordinary differential equations, Am. J. Math. 128 (2006), no. 2, 419-451. $\uparrow 619,622,623,630$

[BRY10] Miriam Briskin, Nina Roytvarf, and Yosef Yomdin, Center conditions at infinity for Abel differential equations, Ann. Math. 172 (2010), no. 1, 437-483. $\uparrow 618,622,627,632,642$

[Che72] Leonid A. Cherkas, On the conditions for the center for certain equations of the form $y y^{\prime}=P(x)+Q(x) y+R(x) y^{2}$, Differ. Uravn 8 (1972), 1435-1439. $\uparrow 638$

[Che77] Kuo-Tsai Chen, Iterated path integrals, Bull. Am. Math. Soc. 83 (1977), no. 5, 831-879. $\uparrow 620$

[Chr99] Colin Christopher, An algebraic approach to the classification of centers in polynomial Liénard systems, J. Math. Anal. Appl. 229 (1999), no. 1, 319-329. ^638

[Chr00] Abel equations: composition conjectures and the model problem, Bull. Lond. Math. Soc. 32 (2000), no. 3, 332-338. $\uparrow 624,625,626$

[CL07] Colin Christopher and Chengzhi Li, Limit cycles of differential equations, Advanced Courses in Mathematics - CRM Barcelona, Birkhäuser, 2007. $\uparrow 638$

[CLN96] Dominique Cerveau and Alcides Lins Neto, Irreducible components of the space of holomorphic foliations of degree two in $\mathbb{C} P(n), n \geqslant 3$, Ann. Math. 143 (1996), no. 3, 577-612. $\uparrow 616$

[Dul08] Henri Dulac, Détermination et intégration d'une certaine classe d'équations différentielles ayant pour point singulier un centre, Bull. Sci. Math. II. Sér. 32 (1908), 230-252. ^616, 635

[FGX16] Jean-Pierre Françoise, Lubomir Gavrilov, and Dongmei Xiao, Hilbert's 16th problem on a period annulus and Nash space of arcs, https://arxiv.org/abs/1610.07582, to appear in Math. Proc. Camb. Philos. Soc., 2016. $\uparrow 635$

[Fra96] Jean-Pierre Françoise, Successive derivatives of a first return map, application to the study of quadratic vector fields, Ergodic Theory Dyn. Syst. 16 (1996), no. 1, 87-96. 625

[Gav05] Lubomir Gavrilov, Higher order Poincaré-Pontryagin functions and iterated path integrals, Ann. Fac. Sci. Toulouse, Math. 14 (2005), no. 4, 663-682. ^620, 626, 633

[GGS19] Jaume Giné, Maite Grau, and Xavier Santallusia, A counterexample to the composition condition conjecture for polynomial abel differential equations, Ergodic Theory Dyn. Syst. 39 (2019), no. 12, 3347-3352. 个619, 623, 642, 645 
[GI09] Lubomir Gavrilov and Iliya D. Iliev, Quadratic perturbations of quadratic codimensionfour centers, J. Math. Anal. Appl. 357 (2009), no. 1, 69-76. ^638

[GM07] Lubomir Gavrilov and Hossein Movasati, The infinitesimal 16th Hilbert problem in dimension zero, Bull. Sci. Math. 131 (2007), no. 3, 242-257. $\uparrow 628$

[GMN09] Lubomir Gavrilov, Hossein Movasati, and Isao Nakai, On the non-persistence of Hamiltonian identity cycles, J. Differ. Equations 246 (2009), no. 7, 2706-2723. $\uparrow 620$

[Hai87] Richard M. Hain, The geometry of the mixed Hodge structure on the fundamental group, Algebraic geometry, Bowdoin, 1985 (Brunswick, Maine, 1985) (Spencer J. Bloch, ed.), vol. 46, Proceedings of Symposia in Pure Mathematics, no. 2, American Mathematical Society, 1987, pp. 247-282. $\uparrow 620$

[Har95] Joe Harris, Algebraic geometry. A first course, Graduate Texts in Mathematics, vol. 133, Springer, 1995, corrected reprint of the 1992 original. $\uparrow 629$

[Ili98] Iliya D. Iliev, On second order bifurcations of limit cycles, J. Lond. Math. Soc. 58 (1998), no. 2, 353-366. $\uparrow 625$

[LN80] Alcides Lins Neto, On the number of solutions of the equation $d x / d t=\sum_{j=0}^{n} a_{j}(t) x^{j}$, $0 \leqslant t \leqslant 1$, for which $x(0)=x(1)$, Invent. Math. 59 (1980), no. 1, 67-76. $\uparrow 625$

[LN14] _ Foliations with a Morse center, J. Singul. 9 (2014), 82-100. $\uparrow 617,635,637$

[Mov04] Hossein Movasati, Center conditions: rigidity of logarithmic differential equations, J. Differ. Equations 197 (2004), no. 1, 197-217. $\uparrow 629,630$

[PM09] Fedor Pakovich and Mikhael Muzychuk, Solution of the polynomial moment problem, Proc. Lond. Math. Soc. 99 (2009), no. 3, 633-657. $\uparrow 625$

[Rou98] Robert Roussarie, Bifurcation of planar vector fields and Hilbert's sixteenth problem, Progress in Mathematics, vol. 164, Birkhäuser, 1998. $\uparrow 625$

[Yom03] Yosef Yomdin, The center problem for the Abel equation, compositions of functions, and moment conditions, Mosc. Math. J. 3 (2003), no. 3, 1167-1195, dedicated to Vladimir Igorevich Arnold on the occasion of his 65th birthday. $\uparrow 624,625,632$

[Zar19] Yadollah Zare, Center conditions: Pull-back of differential equations, Trans. Am. Math. Soc. 372 (2019), no. 5, 3167-3189. $\uparrow 630$

[Żoł94] Henryk Żoładek, Quadratic systems with center and their perturbations, J. Differ. Equations 109 (1994), no. 2, 223-273. $\uparrow 634,635$

Manuscript received on 28th November 2018, revised on 11th August 2019, accepted on 6th November 2019.

Recommended by Editor J. V. Pereira. Published under license CC BY 4.0.

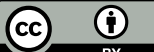

This journal is a member of Centre Mersenne.

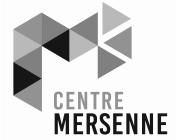

Lubomir GAVRILOV

Institut de Mathématiques de Toulouse

UMR5219 Université de Toulouse

CNRS UPS IMT 
31062 Toulouse Cedex 9 (France)

lubomir.gavrilov@math.univ-toulouse.fr 